\begin{tabular}{|c|c|}
\hline Citation & $\begin{array}{l}\text { Kunal S. Mali, Nicholas Pearce, Steven De Feyter and Neil R. Champness } \\
\text { Frontiers of supramolecular chemistry at solid surfaces } \\
\text { Chem. Soc. Rev., 2017, 46, 2520-2542 }\end{array}$ \\
\hline Archived version & $\begin{array}{l}\text { Author manuscript: the content is identical to the content of the published } \\
\text { paper, but without the final typesetting by the publisher }\end{array}$ \\
\hline Published version & http://dx.doi.org/ 10.1039/C7CS00113D \\
\hline Journal homepage & http://pubs.rsc.org/en/journals/journalissues/cs\#!recentarticles\&adv \\
\hline Author contact & $\underline{\text { steven.defeyter@kuleuven.be }}$ \\
\hline & + $32(0) 16327921$ \\
\hline IR & https://lirias.kuleuven.be/handle/123456789/595956 \\
\hline
\end{tabular}

(article begins on next page) 


\title{
Frontiers of supramolecular chemistry at solid surfaces
}

\author{
Kunal S. Mali, (D) ${ }^{a}$ Nicholas Pearce, ${ }^{b}$ Steven De Feyter (D) *a and \\ Neil R. Champness (D)*b
}

Received 14th February 2017

rsc.li/chem-soc-rev

\begin{abstract}
The application of supramolecular chemistry on solid surfaces represents an exciting field of research that continues to develop in new and unexpected directions. This review highlights recent advances in the field which range from the fundamental aspects of the thermodynamics of self-assembly through to the development of new materials with potential application as new materials. The unique aspects of working on solid surfaces are highlighted and advances in the assembly of many component systems and highly complex fractal-like and quasicrystalline systems discussed. The unique features of working in the surface-based environment and the utilisation of scanning probe microscopies as a primary characterisation tool are highlighted.
\end{abstract}

\section{Introduction}

As we mark the 50th anniversary of the landmark work of Charles Pedersen, ${ }^{1}$ widely regarded as the birth of supramolecular chemistry, the 'chemistry beyond the covalent bond' continues to flourish at an astonishing rate. It has broken barriers of scientific creativity in a diverse array of disciplines including chemistry, biology, physics and material science and has matured as an independent field over the past couple of decades. Besides the innumerable advances in science and technology brought about by supramolecular chemistry, one of the most important and enduring implications of the early

${ }^{a}$ Division of Molecular Imaging and Photonics, Department of Chemistry, $K U$ Leuven - University of Leuven, Celestijnenlaan 200F, B3001 Leuven, Belgium

${ }^{b}$ School of Chemistry, University of Nottingham, Nottingham, NG7 2RD, UK

work of Pedersen, Cram and Lehn is the widespread appreciation of the importance of the non-covalent bond. The intimate relationship between the non-covalent forces that hold molecules together in a material and its bulk properties is extensively acknowledged. Given their ever-emergent nature, the concepts in supramolecular chemistry are rapidly being employed in different fields including pharmaceutical sciences, separation technology, catalysis, chemical and biological sensors, and molecular electronics to name a few. ${ }^{2}$

Supramolecular chemistry has been explored in a diverse range of environments. While a large body of work has been reported for systems studied in the solution phase, ${ }^{2}$ supramolecular chemistry in the solid-state has also evolved significantly over the years and concerns crystal engineering of solids. ${ }^{3}$ Assembling molecules at solid interfaces offers a unique environment for studying supramolecular chemistry.

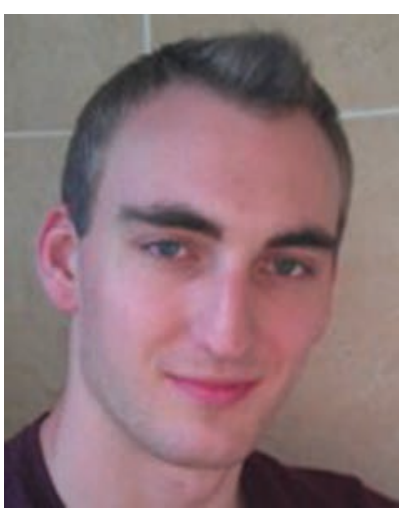

Nicholas Pearce
Nicholas Pearce obtained his PhD in chemistry in 2016 from the University of Nottingham under the supervision of Prof. Neil Champness. His doctoral studies focussed on the synthesis of rylene diimide molecules and dyad systems for application in photoactivated devices. Currently, he is a postdoctoral fellow in the Champness group where his work involves surfacebased self-assembly. scopy. Currently, he is a senior postdoctoral fellow at KU Leuven in the De Feyter group where his research involves different aspects of surface-confined supramolecular self-assembly.
Kunal S. Mali
Kunal S. Mali obtained his PhD in of Mumbai (India) under the supervision of Dr G. B. Dutt. His doctoral work focused on inves tigation of fast dynamic processes in complex media by employing 
1 Surface self-assembly can proceed either via physisorption or chemisorption of molecules. Here we focus on physisorbed selfassembled monolayers where the molecules are adsorbed along a plane parallel to the solid surface. Also known as 'twodimensional supramolecular chemistry', this area of research provides a wealth of information on self-assembled network formation where organic monolayers are typically analysed by an arsenal of advanced surface science techniques. Although working in two dimensions, rather than three, limits the

10 number of possible arrangements, the presence of a solid surface, and thus the additional interfacial interactions, often add to the complexity of the assembly process. This is because, in addition to the molecule-molecule and molecule-solvent interactions, one has to factor in the molecule-substrate and 15 solvent-substrate interactions as well. While this apparent increase in the level of complexity may at first sight appear as a disadvantage, a clear grasp of the interplay between these interactions can prove valuable for the fabrication of complex interfacial architectures with novel properties and function. ${ }^{4}$

Given that the principles of supramolecular chemistry are at the heart of most bottom-up strategies towards nanomaterials, significant research efforts have been directed towards elucidating the principles underlying molecular self-assembly at interfaces. Although a major focus remains on nanomaterials with solid cores, ${ }^{5}$ arguably the most precise way to study supramolecular chemistry is carrying out molecular selfassembly on ultra-flat solid surfaces. ${ }^{6-8}$ Similar to that in the solution phase and the solid state, the basis of self-assembly on solid surfaces is molecular recognition. This is a phenomenon where molecules recognize other molecules or ions and associate with them via non-covalent interactions, such as hydrogen and halogen bonds, van der Waals forces, ion-dipole, dipoledipole and $\pi-\pi$ stacking interactions. This 'handshake' between assembling units is often weaker than the covalent bond but still allows formation of supramolecular nanostructures due to the collective strength of numerous such 'handshakes'.

Supramolecular chemistry at solid surfaces has rapidly gained popularity in the past few decades. This rapid expansion, to some extent, is a result of the advancement of scanning

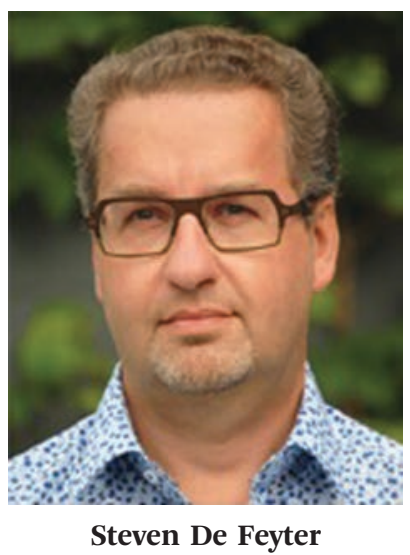

Steven De Feyter is a professor at KU Leuven in Belgium. After completing his PhD with Frans De Schryver at KU Leuven in 1997, he moved for a postdoctoral position to the group of Ahmed Zewail (California Institute of Technology, Pasadena). His research group investigates various aspects of supramolecular chemistry and self-assembly phenomena of surfaces using scanning probe methods with special attention to liquid-solid interfaces.

probe microscopy (SPM). Scanning probe techniques such as atomic force microscopy (AFM), and scanning tunneling microscopy (STM) allow direct visualization of molecular structures adsorbed on solid surfaces. Both STM as well as AFM use sharp probes that raster scan the surface using a piezoelectric device for precise positioning of the probe with respect to the surface. The typical distance between the solid surface and the probe is only a few angstroms and thus atomically flat substrates are preferred. The signal, tunneling current in case of an STM measurement, and force, in case of an AFM, gets modified by nanoscale surface features underneath the tip. While STM imaging works only on conductive surfaces, it is not a necessary condition for substrates used for AFM measurements. Under appropriate experimental conditions, STM and now advanced modes of AFMs, such as non-contact AFM (vide infra) provide sub-molecular resolution of the surface-adsorbed species.

Besides STM and AFM, other surface science techniques also provide insight into nanostructured thin films on solid surfaces. Low-energy electron diffraction (LEED), ${ }^{9}$ grazing-incidence small-angle scattering (GISAS), ${ }^{10}$ and related in-plane X-ray and neutron diffraction techniques ${ }^{11}$ offer structural and mechanistic insights into the formation of thin films. These ensemble measurements however, often provide space-averaged information collected over relatively large areas. Such averaging leads to loss of information on local aberrations. Both STM as well as AFM on the other hand, probe surfaces locally and provide insight into crystalline as well as amorphous structures. The two techniques are extremely versatile and can be used in range of environments such as ultra-high vacuum (UHV), air, water, aqueous electrolytes, organic solvents and gases. The ability to work in a liquid environment means that dynamic processes can also be followed, albeit on relatively slow time scales. Furthermore, they can be operated in temperatures ranging from $\sim 4 \mathrm{~K}$ up to a few hundred Kelvin. Last but not the least, in contrast to the aforementioned techniques, SPM data often provides a direct visual cue to the self-assembled nanostructure and thus has the added advantage of immediate aesthetic appeal.

In this review, we survey recent progress made in the research on surface-supported self-assembled networks. While

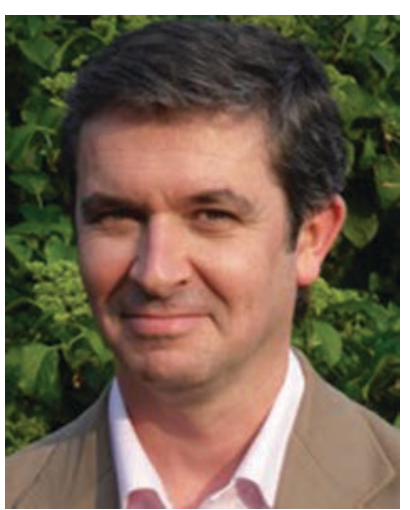

Neil R. Champness
Neil R. Champness is the Professor of Chemical Nanoscience at the University of Nottingham, UK. After completing his $P h D$ at the University of Southampton, UK, with Bill Levason he moved to Nottingham in 1995 reaching his current position in 2004. His research spans chemical nanoscience and all aspects of molecular organization, including surface supramolecular assembly and supramolecular chemistry in the solid-state via crystal engineering. 
1 this review is not comprehensive, we certainly aim to maintain the balance between the width and the depth. The different topics discussed are tied together by the broad theme of interfacial (supramolecular) chemistry. These examples are highlighted since they are related to supramolecular chemistry in liquids, because of the similarities or striking differences. We believe that the discussion touches upon issues that are prerequisites for improved understanding of interfacial chemistry on solid surfaces. The review is structured as follows. The first

10 part contains discussion on the fundamental aspects of interfacial supramolecular chemistry together with a highlight on unusual surface-adsorbed networks based on quasicrystals and Sierpiński triangles. Thermodynamic and kinetic factors controlling the assembly process are discussed. In the second part, different ways of controlling self-assembly are described. The third part surveys reactions at solid interfaces. The emerging fields of 2D covalent organic frameworks (2D-COFs) and chemical functionalization of graphene are discussed. The fourth and the penultimate part highlights recent progress, 0 both on the technical as well as conceptual front, whereas the final section of this review provides some perspectives on future challenges.

\section{2. Fundamental aspects}

Typical supramolecular bonding motifs encountered in solutionphase self-assembly are also commonly found in self-assembled monolayers on surfaces. Translating the information obtained from solution phase to that on surfaces however, is not often straightforward. One has to often apply 'corrections' to the design of self-assembling systems on surfaces and take into consideration the molecule-substrate interactions which are completely absent in solution. These interactions often compete with intermolecular interactions thus altering the outcome of the assembly process. Moreover, molecule-substrate interactions can differ drastically between different substrates.

In a typical experiment, molecules are deposited on to a solid surface either via sublimation or from a solution. The

40 spontaneous assembly proceeds almost instantaneously through a variety of intermolecular and interfacial interactions. As discussed in detail later, this phenomenon is governed by a competition between kinetic and thermodynamic factors. The balance between these factors is drastically different when self作 sidered against that under ambient conditions, especially at the solution-solid interface. Adsorption at the solution-solid interface is often complicated by additional intermolecular and interfacial interactions due to the presence of the solvent which often allows free exchange between molecules already adsorbed onto the surface and those present in the supernatant solution. The absence of solvent and thus the resultant lack of dynamic exchange reduces the relative complexity of self-assembly at the UHV-solid interface.

Whether molecular self-assembly leads to a kinetically trapped or thermodynamically stable equilibrium structure is governed by how rapidly the molecules adsorb and how fast they move on the surface. If the rate of adsorption is faster than the rate of surface diffusion, then molecules are not able to reach the equilibrium structure and are trapped in a diffusionlimited state. On the other hand, if the adsorption rate is slower or comparable to that of surface diffusion, then such a process leads to a thermodynamically favoured equilibrium structure. It was generally assumed that equilibrium processes lead to ordered structures whereas kinetic trapping leads to disorder. However, this view is rapidly changing in light of recent experimental evidence, as discussed in detail later.

\subsection{Ordered yet aperiodic: quasicrystallinity in 2D self- assembly}

Although ordered, periodic structures are often desired, there is increasing interest in creating alternative structures which may possess novel properties. The experimental observation of quasiperiodic crystals in 1982 led to a paradigm shift in the way symmetry in crystals is considered. ${ }^{12}$ A quasicrystalline material consists of an ordered arrangement of atoms or molecules but in an aperiodic fashion. Originally discovered in case of an aluminium-manganese alloy, quasicrystallinity is now confirmed to be present in a number of systems including polymers, liquid crystals, micelles and metallic and silica nanoparticles. While observation of quasicrystalline lattices in self-assembled monolayers is still rare, a few examples have already been reported which illustrate the exotic possibilities available via supramolecular interactions.

A unique example of a quasicrystalline lattice is provided by self-assembly of ferrocenecarboxylic acid (FcCOOH, Fig. 1a) on $\mathrm{Au}(111)$. Upon deposition on the gold surface, FcCOOH selfassembles into cyclic pentamers (Fig. 1b and c). Such a cyclic arrangement is rather unusual given that (mono)carboxylic acids are typically known to form linear assemblies based on hydrogen-bonded dimers. DFT calculations revealed that the pentameric motifs are hydrogen-bonded cyclic catemers (Fig. 1c). Although the arrangement into cyclic catemers is energetically inferior to dimers due to unfavourable bonds angles and entropic reasons, it is possibly stabilized by favourable interactions between the carbonyl group of the carboxyl group on one molecule with the hydrogen atom on the second position of cyclopentadienyl ring of another (Fig. 1d). Hydrogen-bonded dimers are also formed simultaneously however they are dispersed in between the cyclic pentamers. A combination of these dimeric and pentameric motifs imparts quasicrystallinity to the self-assembled network where the domains exhibit local five-fold symmetry and maintain translational and rotational order over short distances. The monolayer however lacks long-range order. The pentagons and their interstitial spaces match the shapes in a so-called Penrose 'P1' tiling (pentagon, rhombus, boat and star, Fig. 1e and f). Fourier transform of STM images revealed local five-fold and ten-fold symmetry of the $\mathrm{FcCOOH}$ monolayer. ${ }^{13}$

Quasicrystallinity has also been discovered recently in the case of metal-organic co-ordination networks formed on $\mathrm{Au}(111)$ surface. ${ }^{14}$ In contrast to the previous example, where 
the network was sustained by hydrogen bonds, here the dominant supramolecular interaction is metal-ligand binding. Coordination of a para-quaterphenyl-dicarbonitrile molecule (qdc, Fig. 2a) with europium ions on $\mathrm{Au}(111)$ surface leads to formation of various co-ordination motifs depending on the stoichiometric ratio between Eu and the organic linker. At a stoichiometric ratio of around 2:5 (Eu:qdc), an open porous network is formed wherein the Eu centres are co-ordinated by four, five or six molecules of qdc. The arrangement of $\mathrm{Eu}$ centers and qdc molecules within this reticulated network appears as a surface tiling pattern made up of randomly distributed squares and triangles. This network closely resema dodecagonal quasicrystal that consists of dodecagonal units separated by randomly distributed square and triangular tiles (Fig. 2b and c). The spontaneous formation and coexistence of surface-confined co-ordination motifs containing four-, five- and six-fold planar coordination nodes (Fig. $2 \mathrm{~d}-\mathrm{g}$ ) is

45 a characteristic feature of this system. Such flexibility arises due to the peculiar metal-organic bonds formed by lanthanides which are predominantly ionic in nature. A second important factor is the nature of the surface. The interactions of the qdc molecules with the gold surface are relatively weak which imparts additional flexibility to the $\mathrm{CN}$ end group during the co-ordination process. Quasiperiodic pattern was not observed when the same system was studied on $\mathrm{Ag}(111)$ which is relatively strongly interacting surface thus highlighting the importance of weaker molecule-substrate interactions. ${ }^{14}$

The quest for obtaining exotic geometric patterns using supramolecular chemistry is not limited to quasicrystals.
Fractal geometry is another type of pattern that is highly sought after. Fractals exhibit a repeating pattern at every scale. The Sierpiński triangle, named after the Polish mathematician Wacław Sierpiński, is a prototypical fractal pattern that exhibits a self-similar geometry at any length scale. The pattern takes the shape of an equilateral triangle which is recursively subdivided into smaller equilateral triangles (Fig. 3b). Fabricating such repetitive patterns has proven to be notoriously difficult and synthetic fractal patterns often show defects.

Fabrication of planar molecular Sierpiński triangles has been reported recently using the tenets of supramolecular chemistry and tools of surface science. 4,4"' -Dibromo$1,1^{\prime}: 3^{\prime}, 1^{\prime \prime}: 4^{\prime \prime}, 1^{\prime \prime \prime}$-quaterphenyl (B4PB, Fig. 3a) - a molecule featuring a $120^{\circ}$ bend in its backbone, was employed as the building block since computational studies have predicted that the shape of the letter ' $\mathrm{V}$ ' is consistent with possible selfassembling Sierpiński triangles. Vapor phase deposition of B4PB followed by fast cooling to $4.4 \mathrm{~K}$ lead to spontaneous self-assembly of defect-free Sierpiński triangles on $\mathrm{Ag}(111)$ surface under UHV conditions. High-resolution STM images reveal that the silver surface is exclusively covered with equilateral triangular features closely resembling the fractal pattern. Sierpiński triangles up to fourth order $(n=4)$ were observed on the surface (Fig. 3b and c), although theoretically the pattern can be infinite. Each node in the assembled network consists of three B4BP molecules closely arranged together in such a way that their $\mathrm{Br}$ termini form a cyclic pattern. The self-assembled network is thus stabilized by weak halogen-halogen interactions and also by weak hydrogen bonding between the $\mathrm{Br}$ atom 
Large-scale STM image of the quasicrystalline metal organic co-ordination network formed on Au(111) surface by depositing Eu and qdc species at $\sim 2: 5$ stoichiometric ratio. Scale bar $=10 \mathrm{~nm}$. The inset shows the 12 -fold symmetric 2D-FFT. Scale bar, $0.84 \mathrm{~nm}^{-1}$. (c) The dodecagonal motifs with a $30^{\circ}$ rotational symmetry: different colours of squares and triangles symbolize distinct orientations with respect to the underlying surface. (d-f) Different metal-organic bonding motifs observed in the quasicrystalline network. The molecular models for the six-fold (3.3.3.3.3.3), five-fold (3.3.4.3.4 or 3.3.3.4.4) and four-fold (4.4.4.4) coordination are provided underneath the digital zooms of STM images. Reproduced from ref. 14 with permission from the Nature Publishing Group. e)
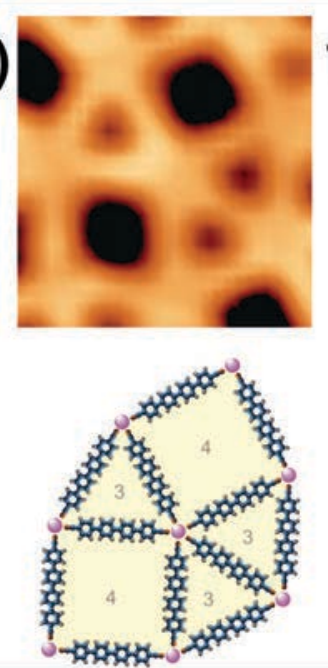

f)
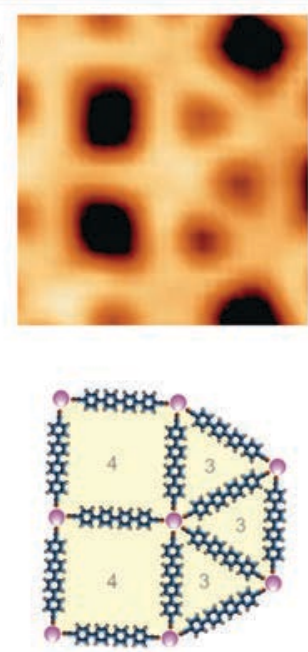

g)
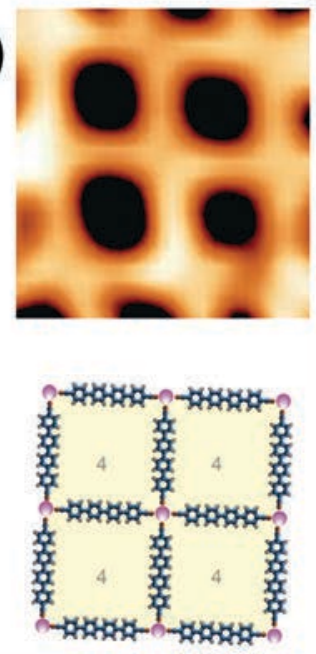

5

10

15

of one B4PB molecule with the $\alpha-\mathrm{H}$ atom of the adjacent B4PB molecule (Fig. 3f). The cyclic arrangement of $\mathrm{Br}$ atoms relative to each other is either clockwise (CW) or counterclockwise 45 (CCW) and thus leads to expression of organizational chirality within the fractal assembly. The CW and CCW triangles are oriented $8^{\circ}$ with respect to each other and $4^{\circ}$ with respect to a reference axis of the $\mathrm{Ag}(111)$ lattice (Fig. $3 \mathrm{~d}$ and e). ${ }^{15}$

The remarkably ordered fractal pattern of B4PB wherein the combination of factors including molecular design, choice of the substrate, precise experimental conditions in terms of temperature control and weak yet directional supramolecular interactions. These factors collectively allow correction errors during the assembly process and reduce the number of defects. Each node in the triangle is precisely defined due to three fold supramolecular binding motif which is also in perfect registry with the underlying lattice of silver. The asymmetric design of the building block ensures minimal defect density. In case a molecule is incorporated into the assembly with its 'wrong' end, the opposite end of the molecule is incapable of forming a stable node. Such defects are eventually removed during the annealing process.

While weak non-covalent interactions were key to the successful formation of Sierpiński triangles in the previous example, it appears that it is not a prerequisite as evident from selfassembly of 1,3,5-tris(4-mercaptophenyl)benzene (TMB, Fig. 4a) on $\mathrm{Au}(111)$ surface $^{16}$ TMB is a three-fold symmetric, thiol functionalized aromatic molecule which forms disordered network after deposition at room temperature. Stepwise annealing of the surface at 200, 250 and $300{ }^{\circ} \mathrm{C}$ caused successive 
d)

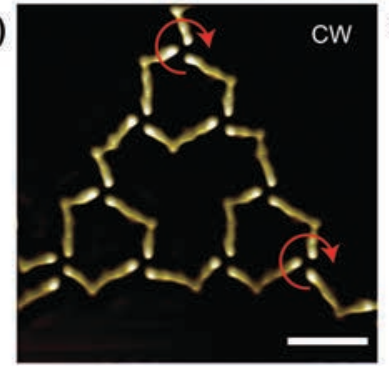

b)

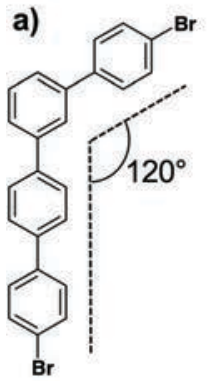

c)
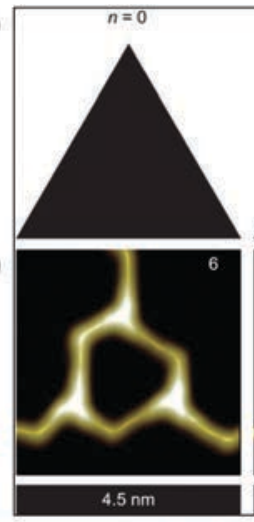
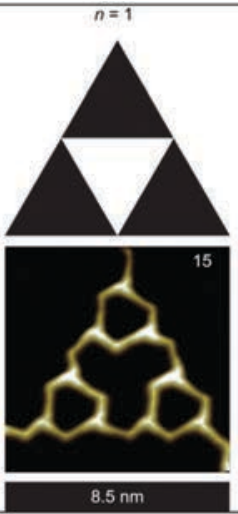
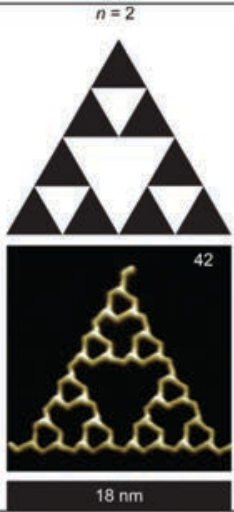

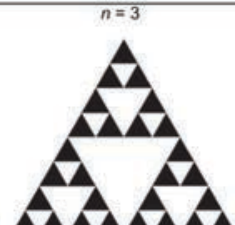

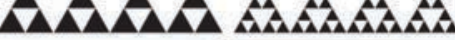
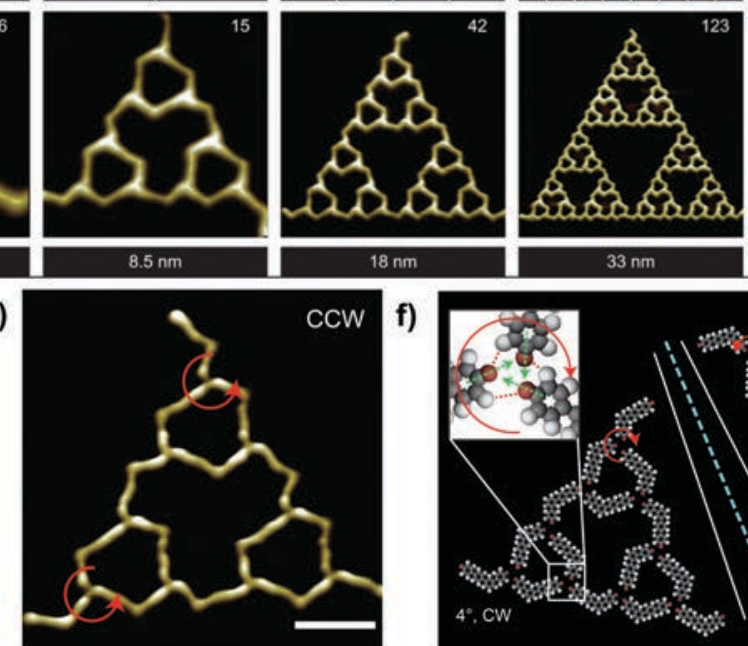

f)

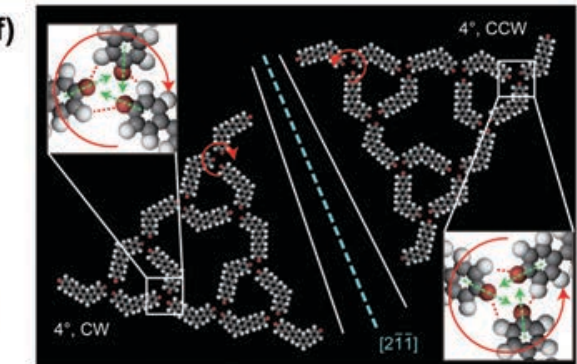

$\mathrm{CCW}$
1

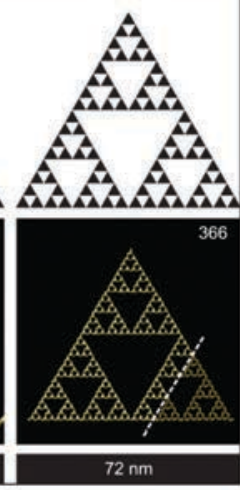

Fig. 3 (a) Molecular structure of B4PB. (b) Simple models of Sierpiński triangles. (c) STM images of self-assembled Sierpiński triangles corresponding to the models provided above. The numbers provided in the upper right corner of each STM image indicate the number of B4PB molecules involved in the assembly. ( $d$ and e) STM images showing enantiomorphous Sierpiński triangles. Scale bar $=2 \mathrm{~nm}$. (f) Molecular models corresponding to STM images provided in panels (d) and (e). Reproduced from ref. 15 with permission from the Nature Publishing Group.

a)

30

35

Fig. 4 (a) Molecular structure of TMB. (b) Large-scale STM image showing the formation triangular aggregates upon heating the heating Au(111) surface at $250{ }^{\circ} \mathrm{C}$. (c) A second generation ( $n=2$ ) Sierpinski triangle. The upper part of the STM image is overlaid with a DFT optimized structure of the covalent first-generation Sierpiński triangle. The lower part shows an overlaid DFT model of a dimer featuring a $\mathrm{C}-\mathrm{S}-\mathrm{C}$ link. Parts (b) and (c) reproduced from ref. 16 with permission from the American Chemical Society.

structural transitions. Gold surface annealed at $200{ }^{\circ} \mathrm{C}$ shows formation of ordered molecular chains. A second structural transition occurs at annealing temperature of $250{ }^{\circ} \mathrm{C}$ leading to formation of triangular aggregates of various sizes. The trianfurther organize into larger self-similar triangles. Sierpiński triangles containing up to 27 molecules $(n=2)$ of TMB were observed. XPS measurements confirmed that the molecular chains observed after annealing at $200{ }^{\circ} \mathrm{C}$ are stabilized by $\mathrm{Au}$-thiolate interactions. The structural transition from chains to Sierpiński triangles however is proposed to have been accompanied by a chemical transformation where the S-Au-S co-ordinate linkages are converted to covalent $\mathrm{C}-\mathrm{S}-\mathrm{C}$ bonds. ${ }^{16}$

The structural design of the two examples of Sierpiński triangles discussed above is fundamentally different. In the triangles based on B4PB molecules, three-fold halogen-halogen and hydrogen bonding interactions gave rise to a triangular topology and the two-fold connection between the triangular nodes is provided by the ' $\mathrm{V}$ '-shaped molecular backbone. In case of TMB however, the three-fold topology is programmed in the molecular structure whereas the ' $\mathrm{V}$ '-shaped $\left(\sim 120^{\circ}\right)$ connectivity is provided by the $\mathrm{C}-\mathrm{S}-\mathrm{C}$ covalent bonds. The 
1 formation of fractal self-assembled patterns requires a powerful combination of molecular design, a mechanism for healing of defects and careful balance of thermodynamic and kinetic factors. Besides the two examples described above, surfacebased fractal self-assembly stabilized by hydrogen-bonding, ${ }^{17}$ metal-organic ${ }^{18,19}$ and covalent bonding ${ }^{20}$ interactions has also been reported. It is notable that in all the examples described above, the Sierpiński triangles were formed under UHV conditions at low-temperatures. The fabrication of fractal selfnetworks under ambient conditions at the solution-solid interface still remains elusive.

\subsection{Kinetics or thermodynamics?}

15 Similar to crystallization of bulk materials, self-assembly on surfaces typically starts with nucleation events that take place at several sites on the surface. Nucleation, which is by default a non-equilibrium process, is followed by growth of nuclei leading to formation of supramolecular domains. The growth process continues till all the available surface area is covered and the domains touch each other producing domain (grain) boundaries. Given the finite surface area, now the domains can only grow at the expense of other domains. But do all these processes occur under thermodynamic equilibrium?

Since the surface patterns formed by a number of organic and metal-organic systems are well-ordered and often defectfree, it was assumed that the self-assembly process occurs at or close to equilibrium conditions. The basic premise behind this assumption was that kinetic assembly would lead to structures with defects and/or disordered patterns and thus crystallinity of the network is an indication that the thermodynamic steady state is reached and that there is a constant exchange between molecules in solution and those on the surface. As mentioned earlier, this view is rapidly changing and it is now widely acknowledged that the self-assembly process at solid surfaces is governed by a competition between kinetic and thermodynamic factors. Their relative contribution depends on the specific experimental conditions used. ${ }^{21}$

The presence of strong kinetic barriers in systems selfassembling on surfaces at room-temperature, at the solutionsolid interface, was recently confirmed. Deposition of 1phenyloctane containing a mixture of cobalt- and nickel octaethylporphyrin (COOEP and NiOEP, Fig. 5a) leads to formation of a mixed monolayer. The two molecules, despite being

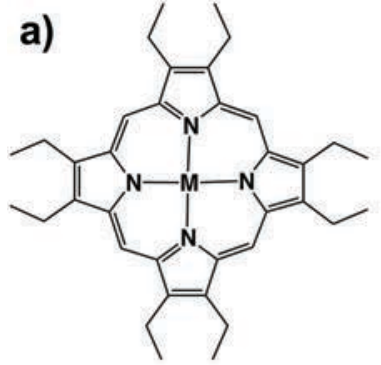

b)

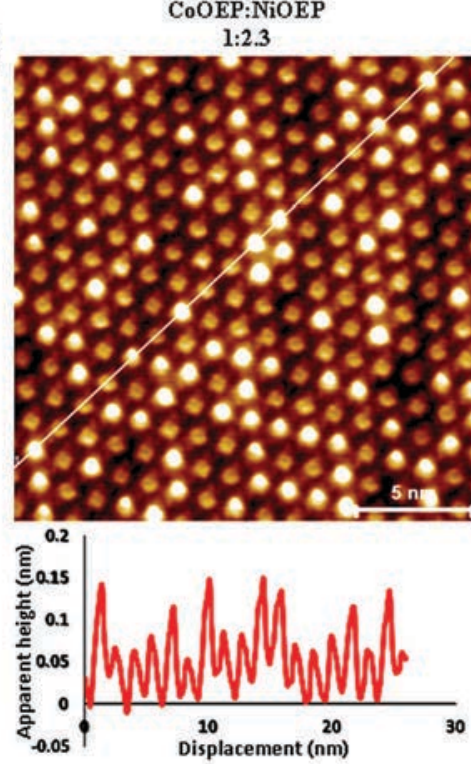

c)

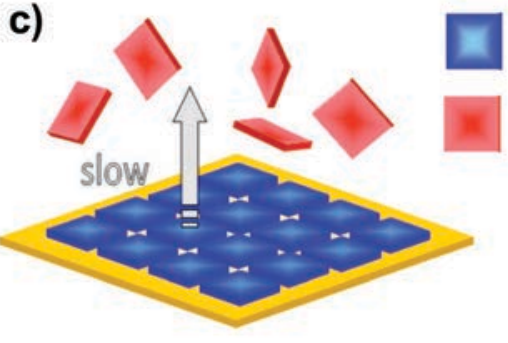

CoOEP:NIOEP 1:3.7
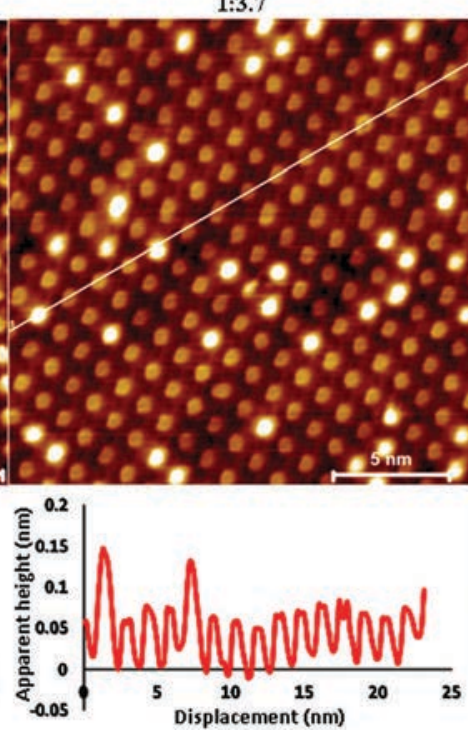

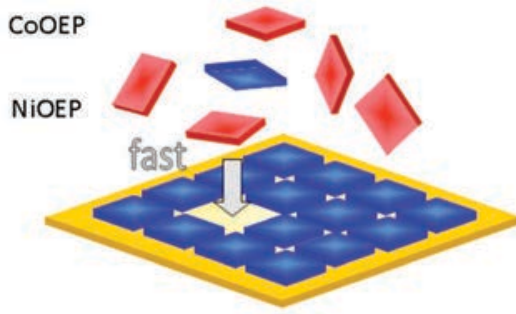

CoOEP:NIOEP 1:7.3
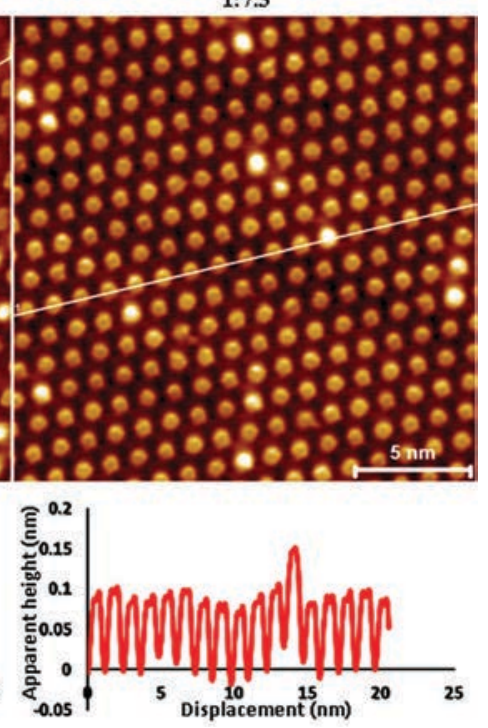

Fig. 5 (a) Molecular structure of metal containing octaethylporphyrin ( $M=\mathrm{Co}$, Ni). (b) STM images of the monolayers formed by varying the relative solution composition of the two porphyrins. The profiles along the white lines shown in the STM image show that the two porphyrin derivatives can be distinguished from their apparent heights. COOEP appears relatively brighter compared to NiOEP. (c) A schematic illustrating the fast adsorption and extremely slow desorption dynamics in this bi-component system. Reproduced from ref. 22 with permission from the American Chemical Society. 
1 structurally similar, can be distinguished in the monolayer due to the peculiar contrast of their metal centers. CoOEP appears brighter in STM images compared to NiOEP (Fig. 5b). STM images obtained by varying the relative solution concentration of the two components (Fig. 5b) revealed that the surface composition i.e. the ratio of the two compounds on the surface, closely reflects the solution composition. The fact that the surface coverage of each component exactly matches its solution mole fraction points to an ideal equilibrium between

10 surface adsorbed molecules and those present in solution. This interpretation, which alludes to identical free energies for the two components in solution as well as surface-adsorbed state, however was found to be inaccurate. ${ }^{22}$

Sequential STM images obtained on the same area revealed

15 that the porphyrin molecules do not desorb once the monolayer is formed at room temperature. Only very little adsorptiondesorption dynamics was observed at elevated temperatures indicating that the adsorption of these porphyrin molecules is entirely controlled by kinetics below $100{ }^{\circ} \mathrm{C}$. These results 0 contradict the conventional wisdom that the self-assembly process at the solution-solid interface is accompanied by adsorption-desorption dynamics that favor formation of crystalline monolayers. Although less strongly bound than covalently-bound thiols, the physisorption of porphyrins on gold is extremely strong with CoOEP showing a desorption rate of only $6.7 \times 10^{-5} \mathrm{~s}^{-1}$ at $135{ }^{\circ} \mathrm{C}$ at the 1-phenyloctane/Au(111) interface. $^{22}$ This example is the extreme form of kinetic control where no desorption occurs and the system remains kinetically trapped over a wide temperature window. The two-component porphyrin system shows similar behavior when assembled on the surface of graphite however desorption occurs faster on the graphite surface compared to that on gold. ${ }^{23}$

The presence of kinetic blockades was also illustrated in a monocomponent system where a single molecule forms two different structural polymorphs but their interconversion is prevented due to kinetic barriers. Dodecyloxy substituted 1,3,5-tristyrylbenzene self-assembles into two different type of structures. A high-density and a low-density porous structure is formed at the 1-phenyloctane/HOPG interface. STM images 40 recorded immediately after application of a droplet of TSB solution onto the HOPG substrate held at $21{ }^{\circ} \mathrm{C}$ revealed only disordered aggregates. This system when allowed to equilibrate for $1 \mathrm{~h}$ at $60{ }^{\circ} \mathrm{C}$ lead to exclusive formation of large domains of the densely packed network. When a droplet containing the 45 same solution of TSB was applied onto HOPG held at $60{ }^{\circ} \mathrm{C}$ and equilibrated for $1 \mathrm{~h}$, exclusive formation of large domains of the porous molecular network was observed. These experiments demonstrate that, for a given final temperature and concentration, the characteristics of the adsorbed monolayer strongly depend on its growth history, that is, on the detailed sequence of temperatures applied during and after the solution droplet deposition. These results also indicate that the nucleation and growth rates for the two polymorphs are different and even at relatively high temperatures, a kinetic blockade prevents the transition of the thermodynamically less stable porous structure to the more stable high-density linear structure. ${ }^{24}$
These recent examples clearly demonstrate that kinetic factors strongly influence the self-assembly at the solutionsolid interface. It must be noted however that the adsorptiondesorption rates also critically depend on the type of assembling molecules, nature of substrate as well as that of solvent and hence the relative contribution of thermodynamic and kinetic factors is expected to vary from system to system. While kinetic blockades may reduce the predictive power over surface self-assembly, it is an aspect that can also be taken advantage of in forming specific phases.

\subsection{Quantitative thermodynamics from microscopy}

The principles of molecular design for self-assembled monolayers often rely on thermodynamic, specifically enthalpic considerations and thus the design strategies work best in conditions where kinetic blockades are either insignificant or completely absent. Given that such conditions are met, it becomes possible to describe the formation of the surfaceconfined patterns not only in a qualitative but also in a quantitative way. Similarly to kinetic parameters, the quantitative estimation of thermodynamic functions that affect selfassembly on surfaces is only emerging in the last few years. ${ }^{21}$

An in-depth understanding of the subtle balance between different enthalpic and entropic contributions is a key towards a complete quantitative thermodynamic description of molecular self-assembly at the solution-solid interface. Conventionally, calorimetry was used to measure adsorption enthalpies of organic molecules adsorbed on graphitic surfaces. In adsorption calorimetry, the heat evolved or absorbed over the course of a chemical process is quantified by measuring the temperature changes in the substrate itself and/or the surroundings. The heat change upon formation of a monolayer is typically small and hence the results are amplified by using large surface-area substrates, such as graphon (graphitized carbon black). Recently, flow microcalorimetry was employed for quantifying adsorption enthalpies of physisorbed self-assembled monolayers formed by different alkylated compounds at the solution-solid interface. It must be noted that the adsorption enthalpies obtained using flow calorimetry include cumulative contributions from solvent-solvent, solvent-substrate, molecule-solvent, molecule-molecule and molecule-substrate interactions. $^{25}$

In order to gain insight into the individual enthalpy contributions from the different intermolecular and interfacial interactions described above, an adapted version of a BornHaber cycle for monolayer self-assembly at the solution-solid interface was developed recently (Fig. 6b).

Self-assembly at the solution-solid interface is driven by the difference in free energy between the molecules adsorbed in the monolayer and their initial state where they are dissolved in solution. In the adapted Born-Haber cycle, the total enthalpy difference between the initial and final states is divided into four enthalpy contributions that are independently accessible via experimental as well as theoretical methods (Fig. 6a). These individual contributions originate from transformations between well-defined reference states. These reference states 


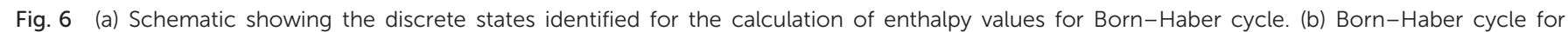

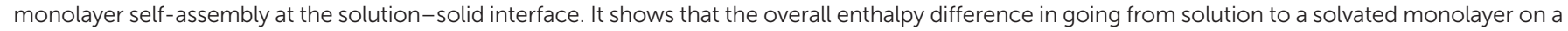

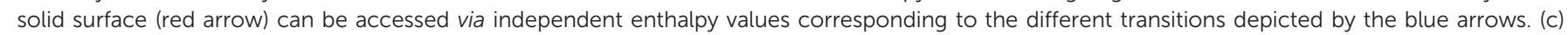

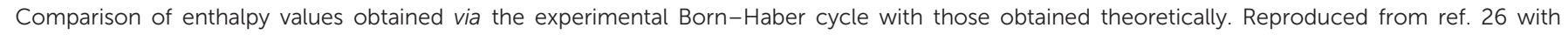
permission from the American Chemical Society.

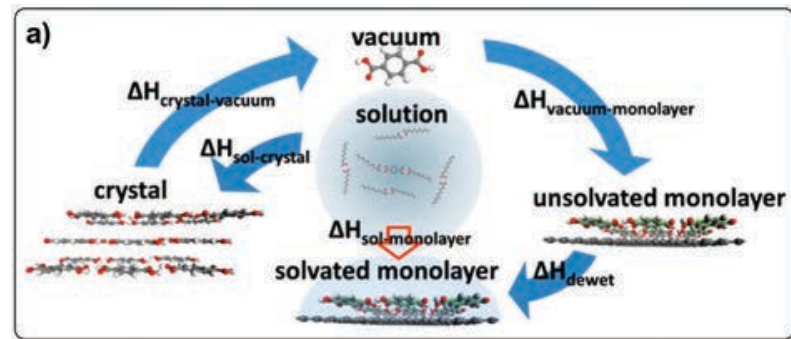

c)

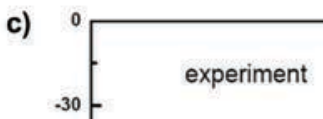

.

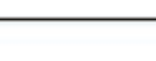

theory
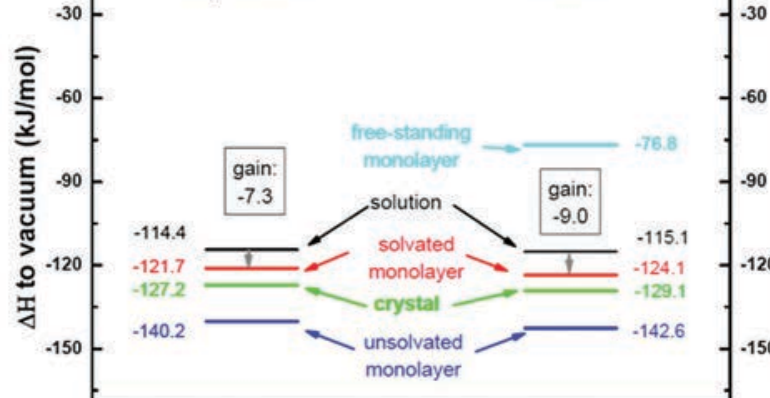

$-90$

$-120$

.150

b)

solution

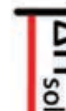

모

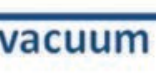
吕 monolayer

\section{unsolvated monolayer}

being the molecules in the bulk crystal, isolated single molecules in vacuum, solvated single molecules and the unsolvated monolayer. Since the solid surface is initially covered by solvent molecules, which are always in excess, one must also consider the heat change upon removal of solvent molecules and subsequent wetting of the monolayer by the solvent. This heat change is accounted for by introducing the dewetting enthalpy term $\Delta H_{\text {dewet }}$. The transitions between the aforementioned states are quantified by the sublimation enthalpy ( $\left.\Delta H_{\text {crystal-vacuum }}\right)$, enthalpy of desorption from the unsolvated monolayer into vacuum $\left(\Delta H_{\text {monolayer-vacuum }}\right)$, the enthalpy of dissolution ( $\Delta H_{\text {crystal-sol }}$ ), and the dewetting enthalpy and the estimation of these quantities allows one to conclude on

The experimental approach for the estimation of the different enthalpies involves a variety of different techniques. Temperature dependent UV-vis absorption measurements performed on a saturated solution of molecule of interest yield the enthalpy of dissolution ( $\left.\Delta H_{\text {crystal-sol }}\right)$. Sublimation enthalpy ( $\left.\Delta H_{\text {crystal-vacuum }}\right)$ is estimated from temperature-dependent measurement of effusion rate of the molecule of interest using a quartz crystal microbalance. The binding enthalpy of the molecules in the monolayer with respect to vacuum ( $\left.\Delta H_{\text {monolayer-vacuum }}\right)$ is obtained from temperature-programmed desorption measurements. Estimation of the dewetting enthalpy term $\left(\Delta H_{\text {dewet }}\right)$ is not straightforward due to uncertainties in the estimation of the enthalpy term related to the wetting of the monolayer by the solvent molecules. Furthermore, contribution of the initial dewetting term is expected to depend on whether or not the solvent molecules form an ordered monolayer on the solid surface. A combination of these independently obtained enthalpies yields the overall enthalpy difference ( $\left.\Delta H_{\text {sol-monolayer }}\right)$. The above-mentioned enthalpy terms can also be estimated using a theoretical approach where molecular mechanics and molecular-dynamics calculations are employed. This BornHaber cycle scheme was first tested for terephthalic acid selfassembly at the nonanoic acid/graphite interface. Comparison of the enthalpy values obtained from the experimental Born-Haber cycle with that obtained theoretically revealed remarkable agreement between the two approaches (Fig. 6c).

The overall enthalpy value obtained using the experimental Born-Haber cycle can be compared with entropy value estimated using a statistical mechanics based approach. ${ }^{27}$ For selfassembly to occur spontaneously, the enthalpic gain must compensate for the entropic cost. The entropic cost becomes higher at lower concentrations. At the concentration threshold of self-assembly, $\Delta G$ should equal 0 and thus $\Delta H=T \Delta S$. The concentration threshold for self-assembly can be estimated in relatively straightforward fashion experimentally and using the enthalpy obtained from the Born-Haber cycle, the entropy cost 
1 at concentration threshold can be quantified. Such comparison revealed that the entropic cost of self-assembly is considerably larger than the enthalpic gain rendering molecular selfassembly on a solid surface thermodynamically unfavorable. However, when the entropic contribution from solvation and dewetting are also considered, the total entropic cost decreases significantly making the self-assembly on surface thermodynamically feasible. ${ }^{26}$

Matsuda et al. developed a cooperative self-assembly model on 10 the basis of the Langmuir adsorption model incorporating two different equilibrium constants, the nucleation constant $K_{\mathrm{n}}$ and the elongation constant $K_{\mathrm{e}}{ }^{28}$ This model successfully reproduced the experimentally observed abrupt change of surface coverage over concentration in a supernatant solution. They carried out STM 15 experiments of a diarylethene derivative at the interface between 1octanoic acid and highly oriented pyrolytic graphite (HOPG). Below a critical concentration, no self-assembled patterns were observed, and above that concentration full surface coverage. In a small concentration window, partial coverage of the surface was observed.

20 Another interesting observation was that more but smaller domains were observed at higher concentrations. A linear relationship was found between the sample concentration and the reciprocal of the average domain size, indicating that the rate of nucleation is proportional to the sample concentration and hence, a large number of domains are generated at a high concentration. The concentration dependence of the fractional coverage suggests a cooperative aggregation on the surface.

In order to gain insight into the enthalpic and entropic contributions of the monolayer self-assembly process, temperature dependent experiments are essential. For instance, Hipps et $a .^{29}$ have reported STM characterization of oxygen binding by the metal centre cobalt(II)octaethylporphyrin physisorbed at the interface between 1-phenyloctane and graphite, and noted that the degree of complexation decreased upon increasing temperature. Binding of oxygen to the porphyrin was a surprise as in solution no oxygen binding occurred. Graphite played a crucial role in facilitating oxygen binding to the metalloporphyrin and temperature dependent STM experiments allows determination of the enthalpic and entropic contributions to the binding process.

\section{Controlling self-assembly pathways}

Surface self-assembly offers a promising opportunity to tailor complex nanoscale architectures. In particular, due to the ability of scanning probe microscopies to identify complex systems at the molecular level, surface supramolecular chemistry allows detailed characterisation of many component selfassembled arrays. This level of characterisation offers distinct advantages over other approaches traditionally employed in solution or solid-state supramolecular chemistry.

\subsection{Multicomponent self-assembly: 2D co-crystallisation}

55 Designing a self-assembled molecular architecture becomes increasingly complex as the number of building blocks used in the system rises. Even single component systems may organise into several different arrangements on the surface environment. For example, trimesic acid (TMA) can organise into two distinct domains, or 2D polymorphs, depending on the hydrogen bonding scheme employed between the TMA molecules. ${ }^{30}$ With such difficulty of control over single component surface networks, three and four component supramolecular systems are consequently rare in the literature. ${ }^{31-33}$ Hostguest interactions are the most common approach to the fabrication of multicomponent $2 \mathrm{D}$ co-crystals, using size and shape discrimination to arrange the guest molecular species in various pores of an underlying host network. ${ }^{31-33}$

Xue and Zimmt were able to avoid the use of host-guest interactions to design a remarkably selective assembly based upon shape complementarity of alkadiyne chains attached to central anthracene units. ${ }^{34}$ The monolayer arising from these substituted anthracene units consists of alternating aryl and aliphatic bars, forming a highly ordered, striped pattern on an HOPG substrate. The aliphatic side chains of the anthracenes segregate their neighbours through two mechanisms: by side chain length and by position of the diyne 'kink' (Fig. 7a). Interdigitation of side chains with complementary kink locations stabilises the structure by optimising van der Waals contacts across a unit cell of 6 diyne-anthracenes and formed the rationale for molecular recognition. Monolayer physisorption on a HOPG surface (Fig. 7b) was found to be especially robust, surviving solvent washing and drying and spanned an area of $10000 \mathrm{~nm}^{2}$.

Two different four-component supramolecular networks have been reported by de Feyter et al. that make use of a guest coronene (COR)-isophthalic acid (ISA) heteromolecular cluster. ${ }^{35,36}$ A peripheral shell of six ISA molecules assemble around the central COR as a cyclic hexamer, ${ }^{37}$ the ISA molecules held together by hydrogen bonds to produce a discrete ISA-COR cluster. In one instance, ${ }^{35}$ the COR-ISA cluster was encapsulated in the hexagonal pore of a Kagomé network formed by interdigitation of the aliphatic arms of a fused dehydrobenzo[12] annulene (DBA) derivative. The fourth molecular species of the 2D co-crystal, triphenylene (TRI) occupied the smaller triangular pores of the Kagomé network.

In the other instance, ${ }^{36}$ a 'core-shell' approach was used to achieve a four component array (Fig. 8), which employs a central molecular template (the core) to organise successive outer concentric molecular layers (the shells). The assembly's third shell was fabricated from a DBA derivative engineered to encapsulate the COR-ISA cluster, whilst itself not forming an extended honeycomb network. As such, a DBA derivative (DBA4OC10) furnished with only four decyloxy chains instead of the usual 6, provided the necessary precision to form efficient van der Waals contacts with the inner ISA shell, without the remainder two decyloxy chains free to interdigitate and consolidate into a larger network. A honeycomb network of DBA bearing six $\mathrm{OC}_{26} \mathrm{H}_{53}$ arms (DBA-6OC26) served as the outermost shell, affording cavities large enough to host the heteromolecular DBA-4OC10-ISA-COR bundle. In isolation, DBA-6OC26 cannot form a porous surface network: the large resultant pores 

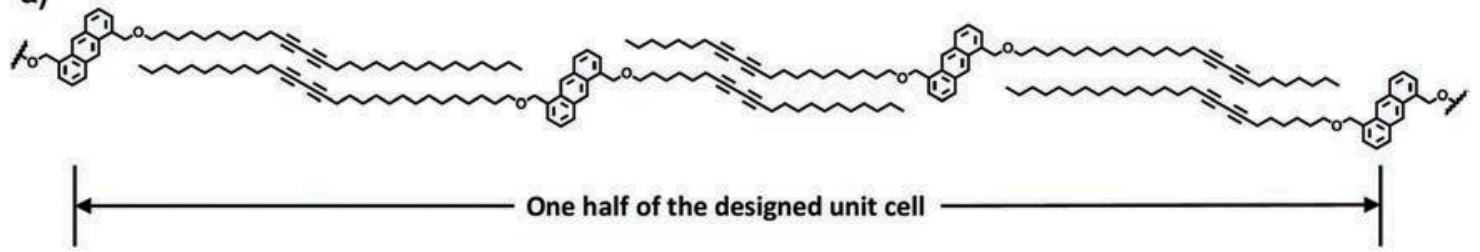

b)
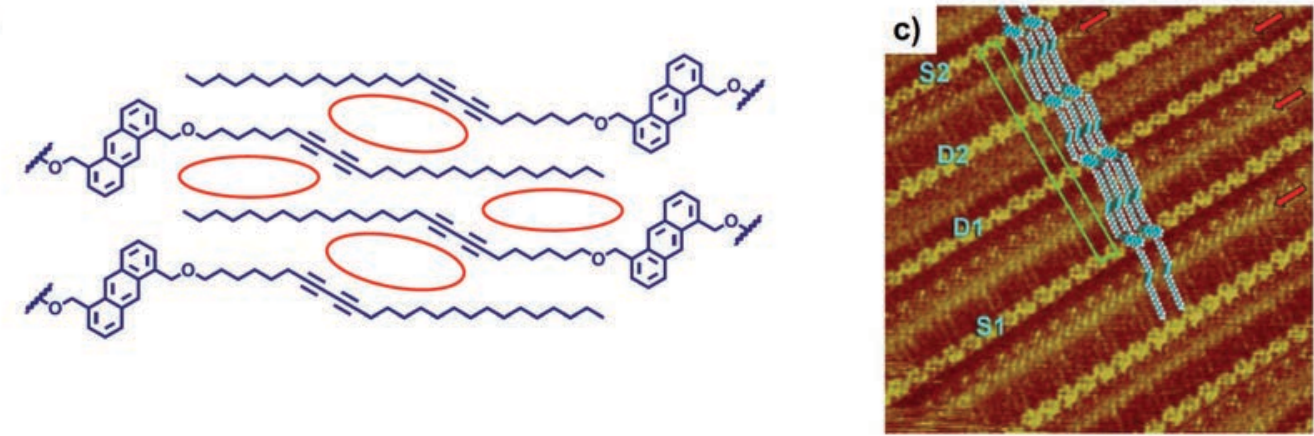

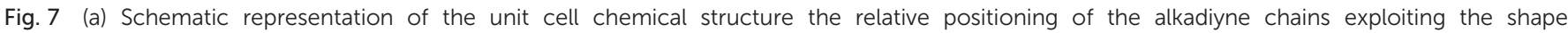
complementarity of the substituted anthracene molecules. (b) Schematic representation of the self-incommensurate nature of the alkadiyne side chains of S2 molecules. Red ovals indicate regions lacking chain-chain van der Waals contact. (c) STM image of the monolayer assembled from a phenyloctane solution of diyne-functionalised molecules showing the relative arrangement of different species S1, D1, D2 and S2 as shown in (a). Red arrows mark diyne columns. Reproduced with permission from the American Chemical Society from ref. 34.

are energetically too costly to stabilise. The presence of the DBA-4OC10-ISA-COR supramolecular cluster was therefore essential to template the formation of the outermost shell.

A ternary self-assembled monolayer of copper phthalocyanine (CuPc), 2,3,7,8,12,13-hexyloxy-truxenone (TrO23) and 1,3,5-tris(10-carboxydecyloxy)benzene (TCDB) that could be tuned to form one of several hierarchical 'flower' type structures was reported by Liu et al. ${ }^{38}$ Tailoring the molar ratio of each component in the solution phase provided control of the level of the hierarchical superstructure obtained on the surface. Each flower was organised into a spiral of petals around a central pore, which displayed organisational chirality. Though no control of overall surface chirality was achieved, domains of enantiopure flowers were observed in roughly equal number.

40 Chiral pores are a common feature observed within molecular monolayers, and recently strategies to resolve the surface into a unique handedness have been reported.

\subsection{Chirality on surfaces}

45 Self-assembly of achiral molecules onto an achiral substrate can induce chirality due to the dimensional constraints of the surface. $^{39,40}$ This resolution of achiral molecules tends to produce a globally racemic surface, divided into many enantiomeric domains and so the development of routes to construct number of approaches have been taken to amplify surfacebased chirality. The 'sergeants and soldiers' strategy is frequently employed to induce chirality of a monolayer, whereby only a small number of chiral sergeant molecules are able to bias the handedness of the supramolecular network formed from achiral soldier molecules. ${ }^{41}$ Once adsorbed onto a surface, achiral DBA molecules can organise into a lattice with hexagonal pores of either clockwise or anticlockwise orientation depending on the alkyl appendage interdigitation pattern. These chiral pores have been demonstrated to serve as enantioselective hosts for chiral DBAs, with clockwise pores operating as hosts for $R$-type DBA enantiomers and anticlockwise nanowells hosting $S$-type DBAs. ${ }^{42}$ Interestingly, the chiral DBA guest molecules primarily function as sergeants for the DBA lattice, ${ }^{43}$ promoting growth of a surface network where the majority of nanowells are the wrong handedness necessary to host the sergeant DBA. ${ }^{42}$ For example, a 30 mol\% solution of an enantiopure DBA (cDBA-OC12 $(S)$ ) and $70 \mathrm{~mol} \%$ achiral DBA-OC12 will form a monolayer with $95 \pm 3 \%$ clockwise nanowells, that are largely unoccupied. The remaining anticlockwise pores, however, show a $60 \pm 4 \%$ occupancy by $\operatorname{cDBA}-\mathrm{OC} 12(S)$ sergeants.

Due to this host-guest complementarity between cDBAOC12 $(S)$ and anticlockwise DBA nanowells, a chiral induction pathway emerges. Upon annealing at $80{ }^{\circ} \mathrm{C}$, the majority handedness is reversed to produce a globally homochiral monolayer of anticlockwise orientation. ${ }^{44}$ This induction process is driven by the guest cDBA-OC12 $(S)$ molecules which have a higher adsorption energy than those acting as sergeants. Upon desorption of the chiral sergeants that define the network handedness, a mass reversal can be observed, due to those guest cDBAs stabilising the anticlockwise pores and nucleating growth of the anticlockwise domains. Contrary to this, if the initial cDBA concentration is too low, such that there are too few guests incorporated into the pores of the initial network, the majority handedness dominates after annealing yielding a surface DBA crystal of exclusively clockwise orientation (Fig. 9). 
Enantiopure sergeant molecules are not always necessary for the formation of a homochiral lattice. The principle of 'majority
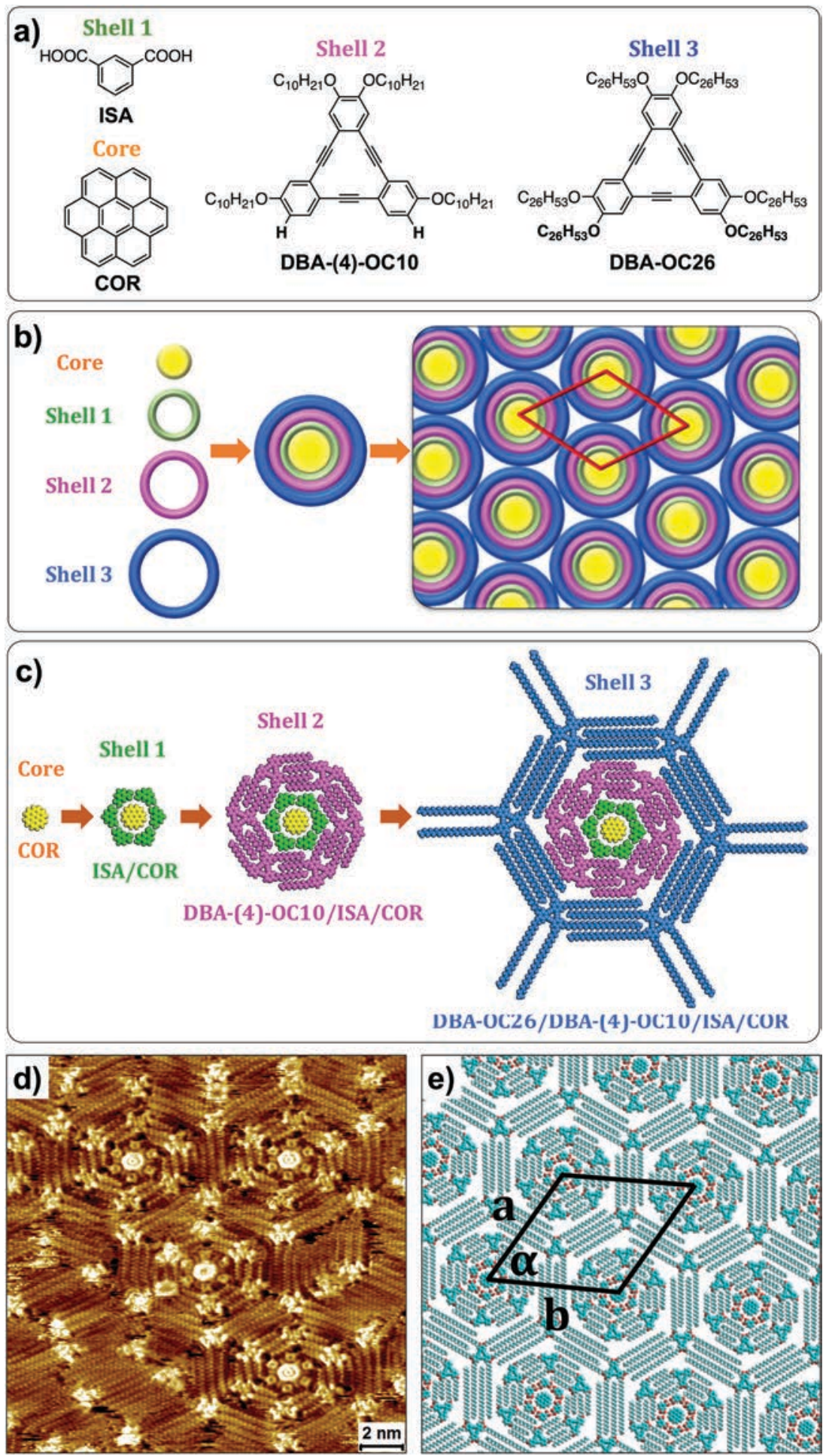

Fig. 8 (a) The molecules used in the four component self-assembly process. (b) Schematic illustration of the 'core-shell' multicomponent system in which each core-shell structure is assembled in a p6 plane group. (c) Molecular models illustrating the design strategy. (d) STM image of the fourcomponent network with three-concentric shells obtained at the octanoic acid/HOPG interface. (e) Molecular model for an ideal, extended network of the four-component system. Reproduced with permission from the Royal Society of Chemistry from ref. 36.

rule' has been validated by the Wan group; demonstrating that a $5.2 \%$ enantiomeric excess of a chiral co-adsorber molecule is sufficient to guarantee global homochirality amongst a 5-(benzyloxy)-isophthalic acid (BIC) derivative network. ${ }^{45}$ Enantiopure $(R)$ - or (S)-2-octanol forms a surface co-crystal with BIC generating entirely clockwise or anticlockwise pores respectively. The 
hydroxyl group of the alcohol directs chirality by forming a tenmembered hydrogen-bonded ring with two acid groups of neighbouring BIC moieties in the supramolecular monolayer. Deposition of a 2-octanol racemate alongside BIC does not selectively produce a homochiral network, although, an unbalanced isomeric mixture shows a distinct preference: when the enantiomeric excess of the (S)-isomer is only $1.3 \%$ the proportion of the BIC network is $68 \pm$ $8 \%$ anticlockwise; increasing to $93 \pm 12 \%$ with $2.6 \%$ enantiomeric excess of the $(S)$-isomer. The BIC network orientation is sustained upon replacement of the 2-octanol molecules with achiral 1,16hexadecanediol forming a global homochiral surface with exclusively achiral building blocks.

A subsequent report investigated the ability of a series of chiral alkyl alcohols to transmit their point stereogenic information to the BIC lattice. ${ }^{46}$ With 2-octanol the stereogenic carbon lies very close to the hydroxyl terminus of the alcohol, though chiral induction is possible even with the stereocentre far away. (S)-6-Methyloctanol, with the chiral carbon located six covalent

40 bonds away from the hydroxyl terminus involved in establishing network chirality, forms BIC architectures of one specific handedness, implying an efficient remote chiral communication. Furthermore, an odd-even effect occurs if the absolute configuration is maintained, but the stereocentre is moved along the alkyl chain, the handedness of the network alternates. Molecular modelling simulations revealed it is energetically favoured for the chiral methyl branch of the co-adsorbing alcohol to orient away from the surface, flipping the surface geometry of the inductive hydroxyl group and thus the network handedness.

\subsection{Stimulus responsive systems}

In addition to the exploitation of molecular design to create specific structures on surfaces ${ }^{32}$ an emerging theme of particular interest is the ability to modify such structures post-assembly. Herein, we will focus on three recent approaches to modifying surface-bound assemblies, heat, light and electric fields.
The use of temperature is an attractive approach to modifying structures, post-assembly. The effect of temperature on the extended structure of a hydrogen-bonded array has been clearly demonstrated by Lackinger et al. ${ }^{47}$ who studied the structures formed by 1,3,5-tris(4-carboxyphenyl)benzene at the solvent/ HOPG interface. Interestingly, the framework formed by this hydrogen-bonding molecule undergoes a phase transition above room temperature. Although such phase transitions have been studied previously, notably in UHV conditions ${ }^{48}$ the study at a liquid/solid interface allows the investigation of solvent dependence. Indeed, the system studied by Lackinger et al. exhibits solvent dependence, even using similar solvents (heptanoic, octanoic and nonanoic acid were used). A reversible phase transition between an open, chicken-wire, hexagonal array and a closed, 'row' structure at the nonanoic acid/HOPG interface is clearly demonstrated by a series of STM images (Fig. 10). The ability to modify the structure of a framework between an open, porous, array and a closed structure with a simple temperature change indicates the possibility for using heating to trigger guest release from supramolecular host structures. A related study ${ }^{49}$ of the self-assembled structures formed by an alkylated dehydrobenzo[12]annulene at a liquid/ solid interface, also illustrates the interconversion between open, hexagonal, phase and a closed phase in which the molecules adopt a close-packed structure. The authors found that both temperature and concentration affect the thermodynamics of the phase transition and successfully describe the importance of enthalpic and entropic effects on the solvation of molecules upon the self-assembly process.

A further method for the post-assembly modification of selfassembled structures is to employ light-initiated processes to make structural changes. A particularly reliable structural change that has received attention is the trans-cis isomerization of azo-benzene moieties. ${ }^{49,50}$ The azo-benzene group is readily integrated into molecules, has extensively studied 


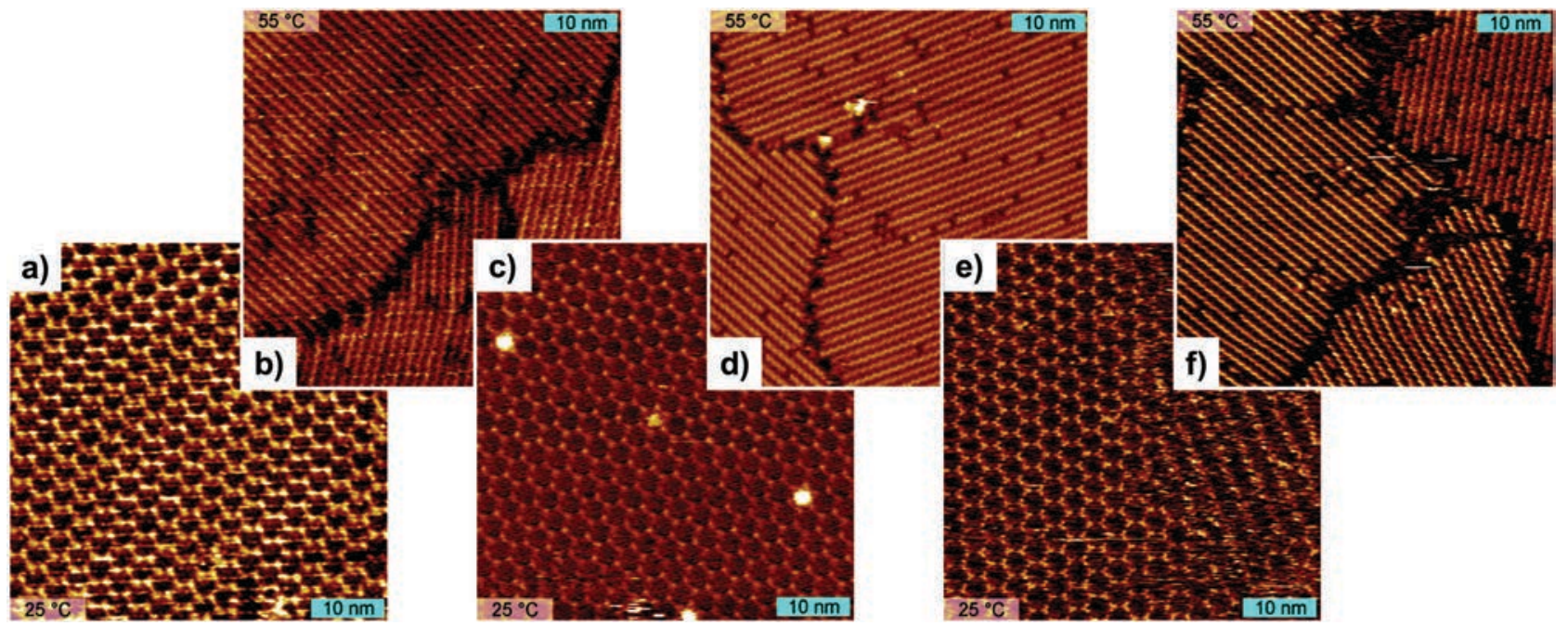

Fig. 10 STM images of the self-assembled arrays formed by 1,3,5-tris(4-carboxyphenyl)benzene at the nonanoic acid/HOPG interface illustrating as acquired during repeated heat-cool cycles of saturated BTB in solutions demonstrating the reversibility of the phase transition between the open, hexagonal, phase at $25^{\circ} \mathrm{C}$ ( $a, c$ and e) and the close-packed, 'row', phase at $55{ }^{\circ} \mathrm{C}(\mathrm{b}, \mathrm{d}$ and f). Reproduced with permission from the American Chemical Society from ref. 47.

photophysical behavior and is therefore a highly attractive target for studies of light-initiated modification strategies. Two studies ${ }^{50,51}$ have employed the azo-benzene isomerization to induce structural changes, modifying pore sizes in open selfassembled structures and therefore altering the 2D structure's ability to act as a host for guest molecules.

Shen et al. ${ }^{50}$ employ a macrocycle that contains four azobenzene groups to prepare a porous 2D array on an HOPG 30 substrate. In its all-trans conformation the macrocycle does not effectively act as a host for the target guest, however upon irradiation with UV light the macrocycle is enlarged by the isomerization of two of the azo-benzene units to form the trans, cis, trans, cis conformation of the macrocycle which is sufficiently open to

35 accommodate two guest coronene molecules (Fig. 11). Upon reisomerisation of the azo-benzene groups in visible light, returning the macrocycle to the all-trans conformation, the coronene is released from the macrocycle. A related process has been used by Tobe et $a{ }^{51}$ The study employs a molecule with a dehydrobenzo-

40 [12]annulene core which is decorated with azo-benzene containing alkyl chains that are terminated with isophthalic acid groups. The molecules self-assemble, employing a range of supramolecular interactions, and creating pores from six isophthalic acid groups that trap guest species, including coronene. ${ }^{35-37}$ Photo-initiated 45 isomerization of the azo-benzene groups leads to modification of the guest-containing pore and altering the ability of the supramolecular array to act as an efficient host.

An alternative photoactive moiety that has been successfully employed in the switching of $2 \mathrm{D}$ surface arrays is the diarylethene group. $^{52}$ Samori, Hecht and co-workers ${ }^{53}$ have studied the interconversion of 1,2-bis(2-methyl-5-(4-octadecyloxycarbonylphenyl)thien-3-yl)cyclopent-1-ene at the liquid/surface interface between 1-phenyloctane and an HOPG substrate. The molecule, designated DAE-o in its open form, undergoes an intramolecular ring-closing reaction, to give DAE-c, when exposed to UV light $(310 \mathrm{~nm})$ which can be reversed using visible light $(530 \mathrm{~nm})$

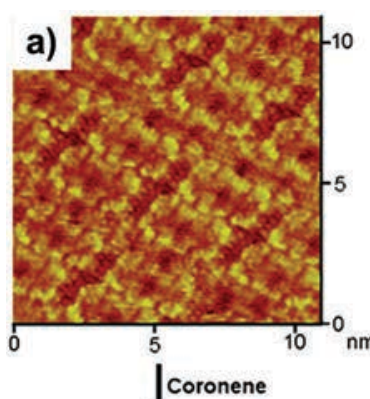

b)
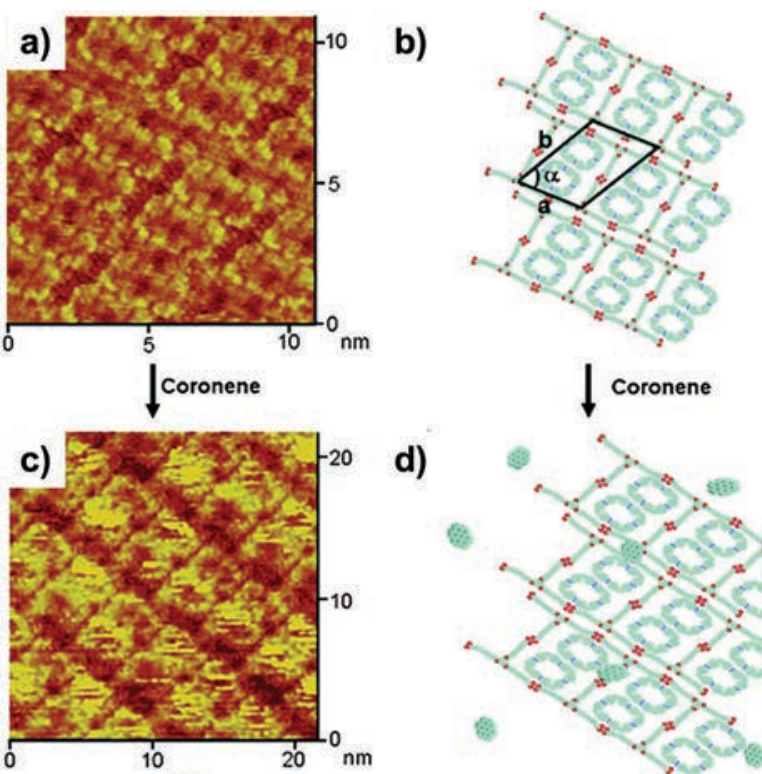

d)
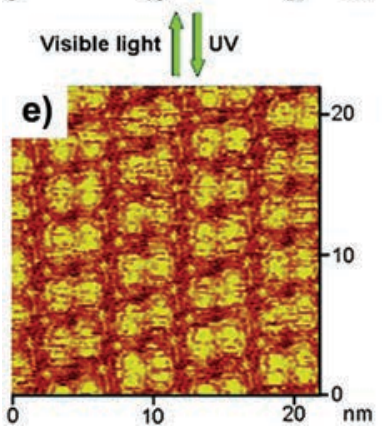

f) Visible light $\hat{\|} \|$ uV

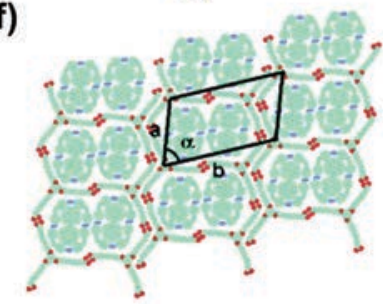

Fig. 11 STM images (a) and model (b) of a tetra-azo-benzene containing macrocycle, co-deposited with a long-chain tricarboxylic acid, (c and d) deposition of coronene onto the macrocycle network with the azobenzenes in an all-trans conformation. Isomerization of the azobenzenes to a trans, cis, trans, cis conformation (e and f) allows encapsulation of two coronene molecules per macrocycle. Reproduced with permission from the American Chemical Society from ref. 50. 
30 (Fig. 12). The interconversion, including formation of a photoirreversible by-product, DAE-b, with an annulated polycyclic core following prolonged UV irradiation, occurs in the solution phase followed by re-adsorption onto the HOPG substrate. Such investigations demonstrate the complexity of systems at the liquid/

35 solid interface with interplay between a number of factors: surface adsorption, solvent/substrate, solvent/molecule interactions coupled with the efficiency of the photo-initiated process. It is clear that there is great scope for using light to initiate postassembly modification of surface supramolecular arrays but that

40 there is a significant need for further studies of the many factors that contribute to their effectiveness.

Another possible external stimulus that can used to influence the self-assembly processes of supramolecular assemblies is electric fields. As STM is perhaps the most widely used tool

45 for the study of such arrays it is evident that the application of an electric field, using the STM tip, is a particularly attractive tool to endeavour to modify and influence the reactivity of molecules on surfaces. The potential for such an approach has been reported by Wan and co-workers. ${ }^{54}$ The authors first assembled a honeycomb array of trimesic acid at an octanoic acid/HOPG interface and then successfully assembled a secondary layer of 1,3,6,8-tetrakis(1-butyl-1 $H$-1,2,3-triazol-4-yl)pyrene on top of the trimesic acid monolayer whilst applying an electric field from the substrate to the STM tip. The pyrene-based molecules assemble into hexagonal, 'flower-like', arrangements, comprising six molecules, as characterized by STM (Fig. 13). In
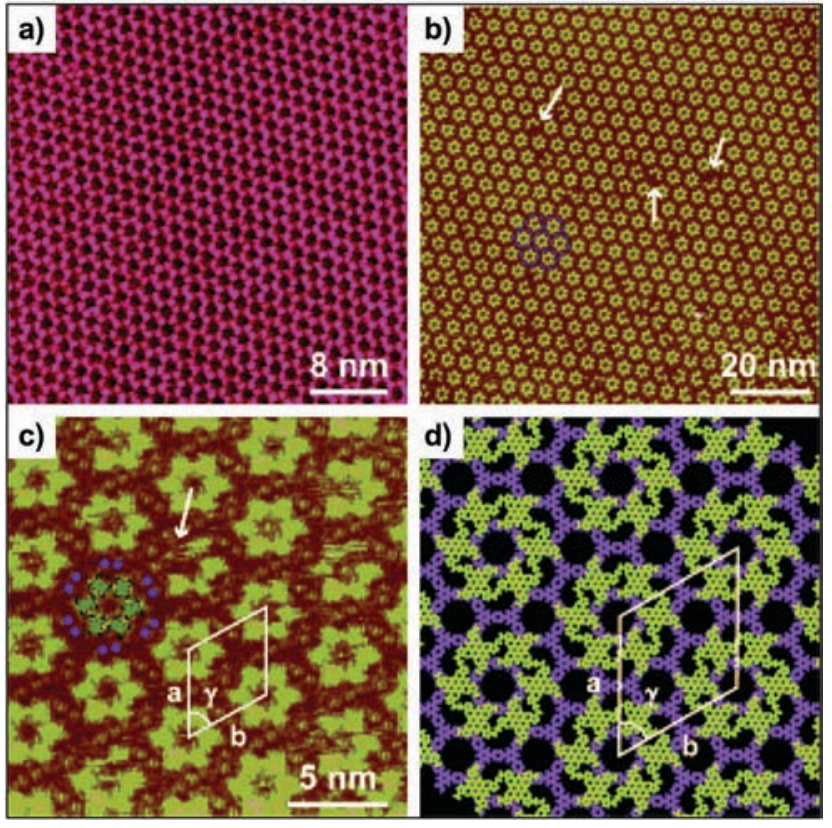

Fig. 13 STM images of (a) the honeycomb structure formed by trimesic acid on HOPG; (b) large scale and (c) high resolution image of the bilayer 'flower' structure formed by 1,3,6,8-tetrakis(1-butyl-1H-1,2,3-triazol-4-yl)pyrene sitting on a trimesic acid monolayer whilst applying an electric field from the substrate to the STM tip. (d) Proposed structural model of the flower structure, 1,3,6,8tetrakis(1-butyl-1H-1,2,3-triazol-4-yl)pyrene - yellow and trimesic acid purple. Reproduced with permission from the Wiley-VCH from ref. 54. 
1 the absence of an applied field a well-ordered Kagome network is formed and apparent height measurements indicate that whereas the 'flower-like' structure adopts a bilayer structure the Kagome lattice is a more common monolayer. Bilayer structures are uncommon in $2 \mathrm{D}$ supramolecular chemistry. ${ }^{55}$ Thus it can be seen that the applied field has a significant effect on the ordering, and even the dimensionality of the selfassembled structure, indeed the bilayer structure is lost when the electric field is removed, the structure reverting to the Kagome arrangement.

Cometto et al. have studied the influence of an applied electric field on the assembly of 1,3,5-tris(4-carboxyphenyl)benzene at the nonanoic acid/HOPG interface. ${ }^{56}$ The same system has been studied extensively by Lackinger et al., ${ }^{57}$ as discussed above and 15 it was found that the system undergoes a phase transition between an open, hexagonal, phased and a close-packed 'row' phase. The phase transition was found to be induced by heating but that the temperature of this transition was solvent dependent. This phase transition can also be induced by an electric field. ${ }^{29}$ Thus, applica-

20 tion of a negative potential between substrate and STM tip favors adoption of the open, hexagonal, phase where as a positive potential favors the closed, 'row', phase. The complexity of what at first appears to be a relatively simple hydrogen-bonded system perfectly illustrates the many factors that contribute to the formation of self-assembled structures at surfaces, in this case including temperature, electric field and solvent effects.

The ability to induce modifications of self-assembled structures and importantly the ability to monitor such processes at the molecular level, using scanning probe microscopy, is a key 30 feature of surface supramolecular chemistry. Although, many approaches exist to monitoring and investigating reactions in supramolecular chemistry it is rare that such studies evaluate modifications at the molecular level. Scanning probe microscopy enables such studies and can lead to remarkable observations of subtle forces that affect assembly processes, even to the extent of gaining insight into reactions at the molecular level. ${ }^{50-54,56,57}$

\section{4. Reactions on surface}

\subsection{Pre-assembly followed by covalent bond formation}

Reactions on surfaces are clearly attractive for creating robust structures but can also be used for the preparation of extended has enjoyed investigation of a period of time is the polymerisation of diacetylene to prepare polydiacetylenes. ${ }^{58} 10,12$ Nonacosadiynoic acid self-assembles on HOPG such that the long chain organic substituents are aligned with the diacetylene moieties in adjacent positions suitable for polymerisation. The polymerisation step can be initiated by either irradiation with UV-visible light or through stimulation by pulsed bias voltage from an STM tip. Recent studies ${ }^{59,60}$ have demonstrated that it is possible to couple such polydiacetylene species to other molecules providing a pathway to exploiting the fabrication of these nanoscale wires. Co-deposition of the diacetylene precursor and either phthalocyanine or $\mathrm{C}_{60}$ results in the formation of 'islands' of the aromatic species sitting in a 'sea' of the diacetylene species. Subsequent polymerization of the diacetylene leads to not only formation of polydiacetylene wires, clearly observed by STM (Fig. 14) but also initiates reaction between the polydiacetylene and the guest phthalocyanine or $\mathrm{C}_{60}$ at the edge of the islands. Thus, polydiacetylene chains are terminated by the aromatic species indicating that wires have been successfully formed. It is believed that the process relies upon the formation of covalent bonds between the growing polydiacetylene chain, which contains a reactive carbene on its termini, and the aromatic species. The studies are particularly important for research targeting the preparation of molecular scale electronic circuitry.

Alkyne-alkyne coupling is a theme that has also been developed by Colazzo et al. ${ }^{61}$ who, rather than focussing on the coupling of diacetylenes, studied the reaction of terminal
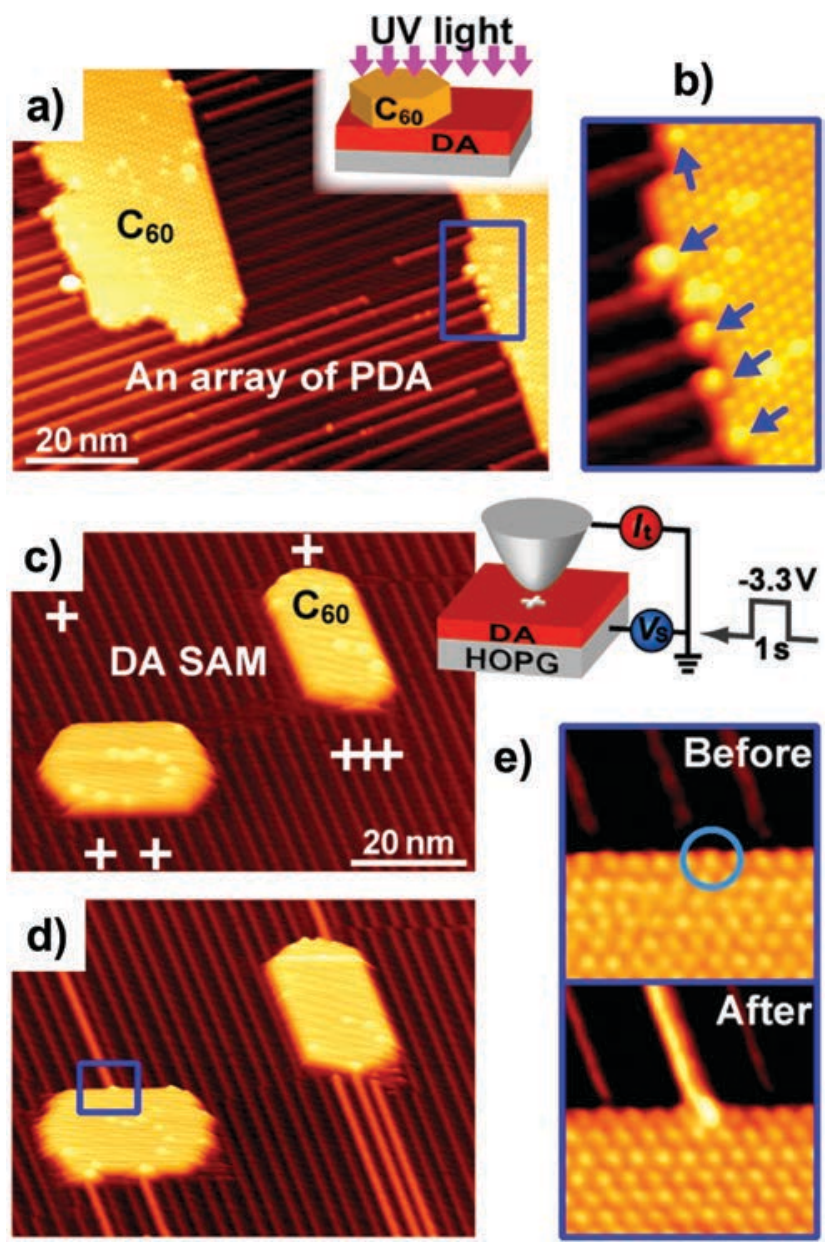

Fig. 14 Creation of junctions between $\mathrm{C}_{60}$ nanoislands and polydiacetylene nanowires. (a) Large scale STM image following UV-induced diacetylene chain and (b) magnified image of the region within the blue box in (a). (c and d) STM images taken before and after inducing chain polymerization by applying voltage at the positions indicated by crosses. (e) magnified image of the region within the blue box in (d) showing the connection between the polydiacetylene nanowire and $\mathrm{C}_{60}$. Reproduced with permission from the American Chemical Society from ref. 60 . 
1 aryl-alkynes to prepare a diacetylene. Although the coupling of terminal alkynes to generate diacetylenes has been known for a number of years, ${ }^{62}$ such reactions have been achieved by heating the precursor molecules on metal substrates to initiate the reaction. Although this process is effective it can lead to unwanted side products. In contrast the study by Colazzo et $a .^{61}$ is an effective way to produce diacetylenes using light to induce the reaction between molecules adsorbed on an HOPG substrate. By using 4-ethynylbenzoic acid the authors

10 are able to use inter carboxylic acid hydrogen-bonding to organise the precursor molecules prior to photo-induced reaction of the acetylene moieties. The approach is highly effective, leading to large scale conversion whilst avoiding unwanted side-products.

15

\subsection{Equilibrium reactions}

Developing covalently-coupled frameworks (typically called covalent-organic frameworks or COFs) on surfaces is an attractive target for research with an anticipated increase in robust-

20 ness in comparison to arrays held together by weaker interactions. ${ }^{63}$ However, methods to prepare covalently coupled arrays can be limited by a need to employ a surface that catalyses the formation of a specific covalent bond and by the formation of arrays with small domain sizes and a high degree of defects. This is in contrast to some supramolecular arrays which can be assembled on a range of substrates and with a high degree of ordering. The difference between the two approaches primarily relates to the irreversible formation of many covalent bonds, the feature which gives rise to their robust nature simultaneously leading to the deficiencies of such structures.

Efforts have been made to overcome this problem by the use of coupling reactions that are not dependent on the nature of the substrate and, due to their reversible character under 5 specific conditions, can lead to large arrays with small proportions of defects. Such coupling processes include condensation reactions such as boronic acid cyclo-condensation, ${ }^{64,65}$ boronate ester formation ${ }^{66}$ and Schiff-base ${ }^{67}$ reactions. Each of these reactions are reversible in nature with the bonds being broken by reaction with water and reformed through a condensation reaction. The formation of boronate ester arrays on graphite substrates has been successfully demonstrated. Lackinger et al. found ${ }^{64,65}$ that whilst arrays with a small domain size are formed during an initial deposition process it is 45 possible to induce an on-surface ripening process to generate arrays with large domain sizes and relatively small number of defects. As the cyclo-condensation of boronic acids can be reversed by water, the presence of water is required for the ripening process to occur, ultimately leading to larger $\mathrm{COF}$ study of 1,4-benzenediboronic acid, ${ }^{64}$ to related, extended analogues using an isoreticular approach. ${ }^{65}$ By increasing the number of phenylene group linking terminal boronic acids, from one phenylene in 1,4-benzenediboronic acid to four phenylenes in quaterphenyldiboronic acid, and by employing the ripening approach, the authors were able prepare COFs
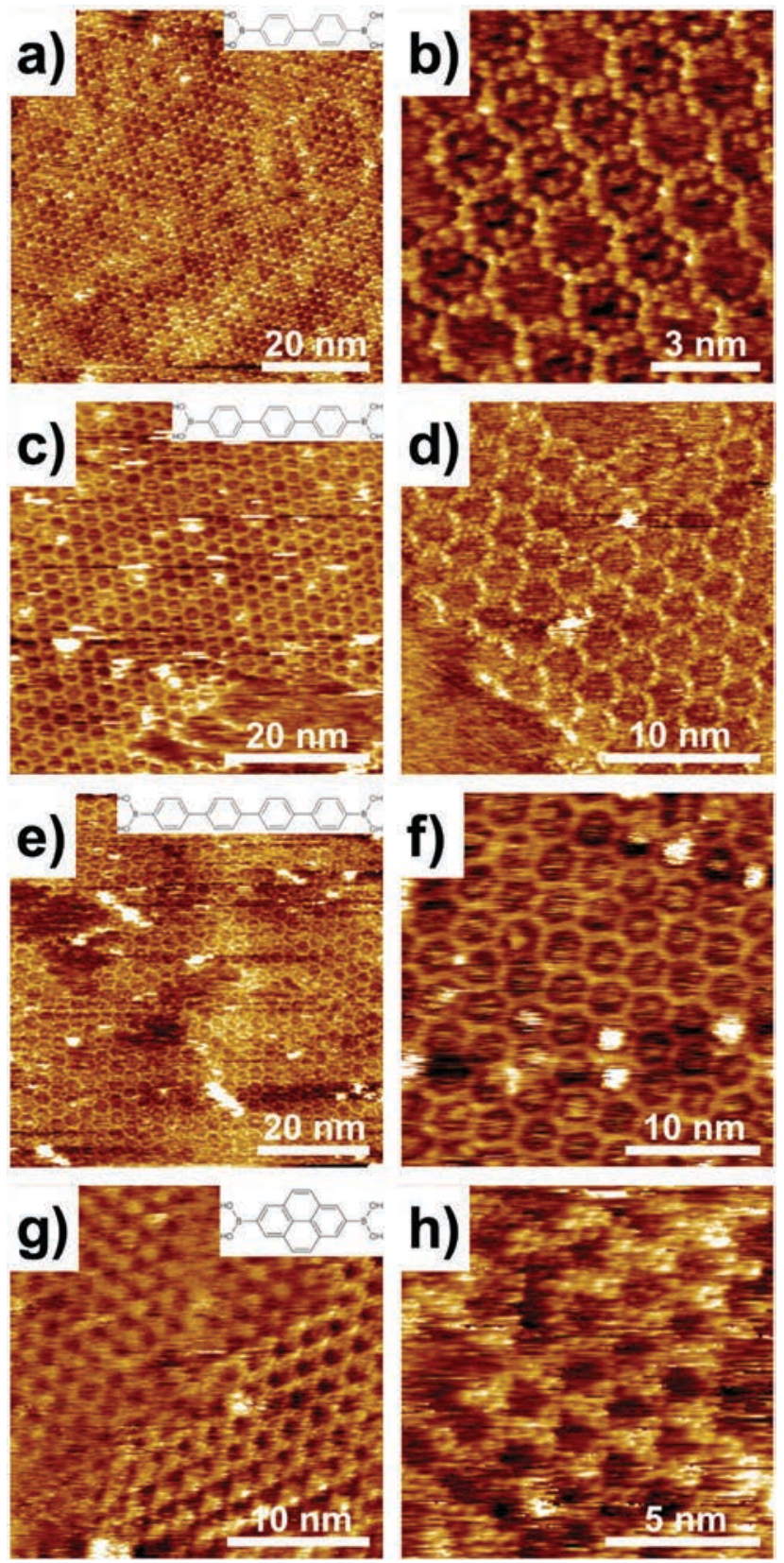

Fig. 15 STM images of 2D COFs prepared by the derived from cyclocondensation of the precursor diboronic acids shown. Note the systematic variation of the hexagonal pore dimensions. Reproduced with permission from the American Chemical Society from ref. 65.

with varying size hexagonal pores (Fig. 15). The study additionally demonstrates that, for certain examples, it is possible to elucidate the supramolecular structure formed by the precursor diboronic acids prior to thermal treatment to initiate the formation of the COF structure.

Wang and co-workers have demonstrated a related approach which employs the reversible behaviour of boronate esters. ${ }^{66}$ The main difference in the strategies are that whereas the cyclocondensation reactions pursued by Lackinger et al. require a single, diboronic acid, precursor the formation of boronate esters requires the reaction of a boronic acid with a diol catechol
10

30

1

5 


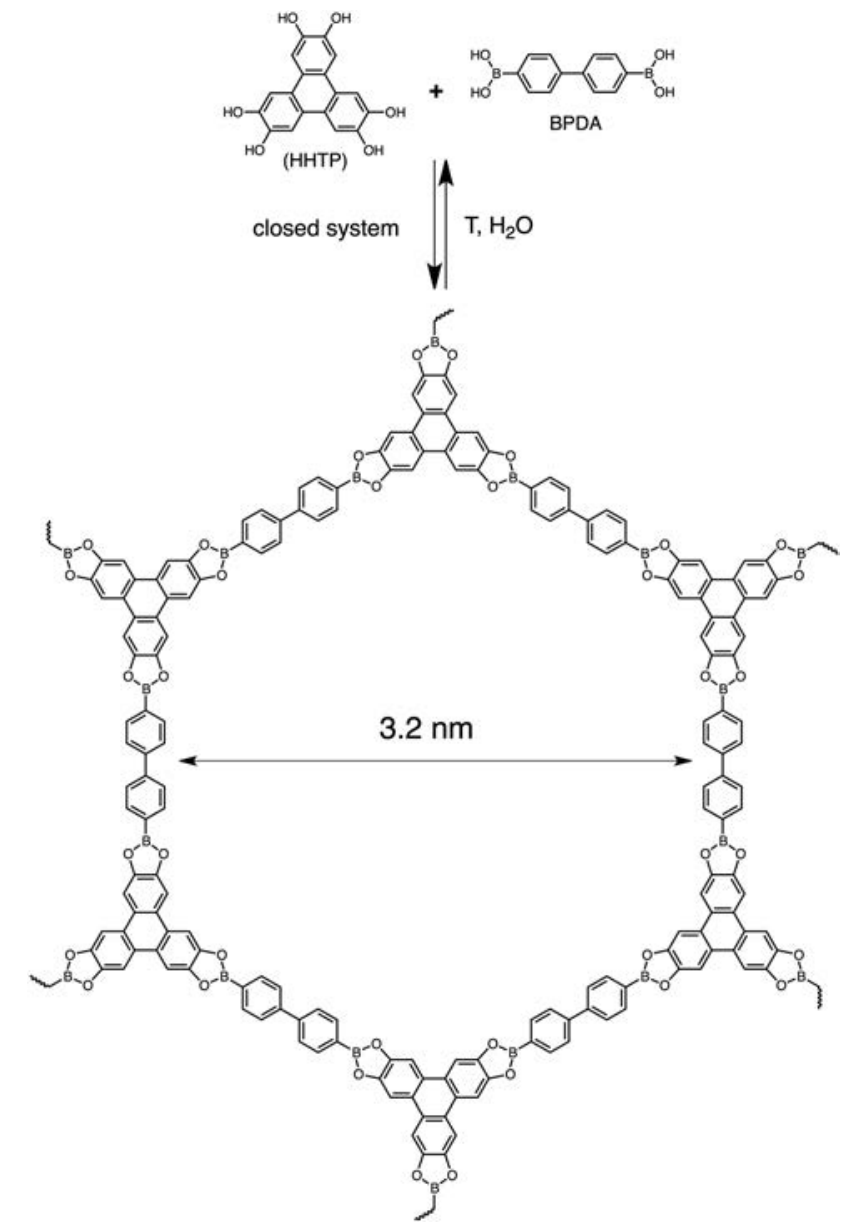

Fig. 16 Schematic representation of the condensation reaction employed by Wang and co-workers to prepare boronate ester based COFs. Reproduced with permission from the Royal Society of Chemistry from ref. 66

species. Thus, the boronate ester approach employs two components and therefore has potential for a greater diversity of arrays. In the example reported 1,4-benzenediboronic acid and 2,3,6,7,10,11-hexahydroxytriphenylene are reacted on an HOPG substrate to form a $2 \mathrm{D}$ hexagonal $\mathrm{COF}$ with pores with a diameter of $3.2 \mathrm{~nm}$ (Fig. 16). As with the cyclo-condensation reactions the reversibility of the boronate ester formation is essential to generate large domain arrays (ca. $100 \mathrm{~nm} \times$ $100 \mathrm{~nm}$ ). The authors achieve the formation of large domains by reacting the starting materials deposited on the HOPG substrate in an autoclave at elevated temperatures and in the presence of a $\mathrm{CuSO}_{4} \cdot 5 \mathrm{H}_{2} \mathrm{O}$ powder. It is hypothesised that the $\mathrm{CuSO}_{4} \cdot 5 \mathrm{H}_{2} \mathrm{O}$ plays a role in regulating the reaction equilibrium through interactions between water molecules and the $\mathrm{Cu}(\mathrm{II})$ salt. ${ }^{67}$

An alternative reaction that reversibly forms covalent bonds is the Schiff-base condensation reaction which involves the reaction of aldehydes with amines. ${ }^{68}$ As with the boronate ester reaction discussed above, ${ }^{66}$ the Schiff-base reaction employs two components and Lei and co-workers have demonstrated the adaptability of the approach through studying the reaction of benzene-1,3,5-tricarboxaldehyde with four different aromatic diamines of varying length and steric properties. As with the boronic acid reactions, the Schiff-base reaction is an equilibrium which involves a dehydration/hydration process and this is effectively employed to prepare large domain arrays on HOPG substrates in the presence of an octanoic acid solvent. The authors also successfully demonstrated that the reaction proceeds at the gas/solid interface under reduced pressure and elevated temperatures. The low vacuum effectively removes solvent molecules but also excess components of the arrays leading to enhanced array crystallinity.

\subsection{Reactions with surfaces: diazonium chemistry on graphite/graphene}

In addition to the in-plane reactions where molecules react with each other (often) along the plane parallel to the solid surface, there is an increasing interest in reaction protocols where the molecules react with the solid substrate itself. This approach has received significant attention in the recent past largely due to importance attached to covalent modification of graphene ${ }^{69}$ and related 2D materials such as $\operatorname{MoS}_{2} \cdot{ }^{70}$ Covalent attachment of small organic molecules to solid surfaces is beneficial from two perspectives. Firstly, it increases the robustness of organic thin films attached onto the solid surface and secondly, the covalent modification of technologically relevant surfaces is known to alter their properties. For example, covalent attachment of small organic molecules to graphene can transform the pristine material, which is known to be chemically insensitive and difficult to disperse in typical organic solvent, into a chemically sensitive, soluble material. In this section we review a small yet significant section of surface covalent modification strategies, namely diazonium chemistry on graphite and graphene. While the examples discussed here do not necessarily involve supramolecular chemistry, they are an important part of the broader field of interfacial chemistry and surface science.

The covalent modification of graphene is an intensively researched area and is often perceived as a relatively robust approach towards graphene functionalization, the other approach being functionalization via physisorption. ${ }^{71}$ One of the most popular reactions employed for modification of graphene is based on diazonium salts. The covalent chemistry involving the attachment of aromatic diazonium salts on carbon surfaces has been known since the 1990s. The reaction mechanism involves the transfer of an electron from graphene to the aryl diazonium cation, which converts the latter into an aryl radical with the loss of nitrogen. The radical then attacks the $\mathrm{sp}^{2}$-hybridized carbon of the graphene lattice thereby covalently attaching itself onto the basal plane (Fig. 17a).

A combination of scanning probe microscopy and Raman spectroscopy can provide valuable information on the degree of covalent modification of graphitic substrates. Electrochemical reduction of 4-nitrobenzenediazonium (4-NBD) tetrafluoroborate enables covalent attachment of 4-nitrophenyl radicals to the graphite surface. This system however suffers from a sidereaction where, besides attaching onto the graphite surface, the 
1 4-nitrophenyl radicals also attack the 4-nitrophenyl groups already attached to the surface. This leads to formation of oligomers thereby reducing the density of grafted aryl groups and increasing the thickness of the resultant covalent film (Fig. 17b). This shortcoming, however, can be overcome by employing 3,5-bis-tert-butyl-benzenediazonium (3,5-TBD) salt which has sterically hindering tert-butyl groups that inhibit the side reaction thereby limiting the thickness of the layer to a monolayer (Fig. 17b). Detailed concentration variation of the diazonium reagent then allows precise control over the density of grafted aryl groups (Fig. 17c and d). The evolution in the morphology of the surface was followed using AFM and STM whereas concomitant changes in graphite lattice were evaluated using Raman spectroscopy (Fig. 17e). Raman spectroscopy has been an integral part of graphene research any covalent or non-covalent modification of graphene changes its unique Raman spectrum. The covalent attachment of aryl radicals onto the graphite/graphene surface proceeds via $\mathrm{sp}^{2}$ to $\mathrm{sp}^{3}$ hybridization. The generation of these $\mathrm{sp}^{3}$-hybridized defects is associated with the appearance of a characteristic D-band at around $1350 \mathrm{~cm}^{-1}$ together with changes in $\mathrm{G}$ and $2 \mathrm{D}$ bands in the Raman spectrum of graphene. Monitoring of the surface morphology vis-àvis the changes in the Raman spectra provides a complete picture of the surface modification process. ${ }^{72}$
One of the unique aspects of the STM characterization of covalently modified graphite surfaces is that the attached aryl groups can be detached from the surface using the STM tip. This is another example that demonstrates that STM can be used not only for imaging but it can also serve as a nanomanipulation technique. Using suitable tunneling parameters it is possible to remove well-defined patches of the grafted material thus providing an equivalent of STM nanolithography. Furthermore, the detachment proceeds with rehybridization of the surface lattice defect from $\mathrm{sp}^{3}$ back to $\mathrm{sp}^{2}$ as demonstrated by combined scanning probe and Raman measurements (Fig. 17fh). While the precise mechanism of this rehybridization is still under scrutiny, the ability to produce nanometer-wide, welldefined patterns of pristine graphite/graphene within the 'forest' of grafted aryls is exciting, facilitating the use of such welldefined regions to study self-assembling systems under (further) lateral confinement (Fig. 17i). Initial studies within such so-called 'nanocorrals' with precise size, shape and orientation have already provided some interesting insights into the self-assembly processes under nanoconfinement. The probability of self-assembly within such confined regions is reduced significantly, when the size of the nanocorral is smaller than a certain critical size. It is widely acknowledged that scanning a)

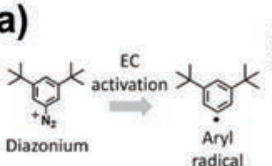

b)
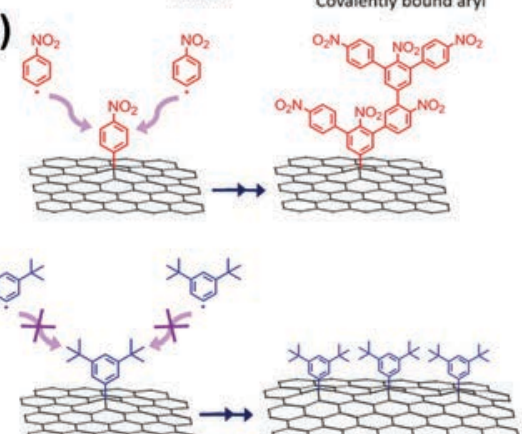

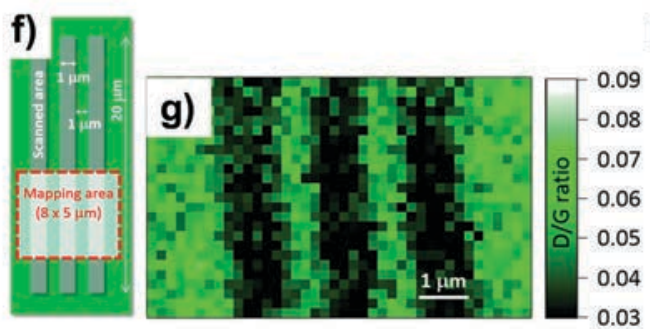

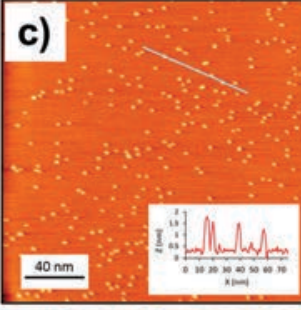

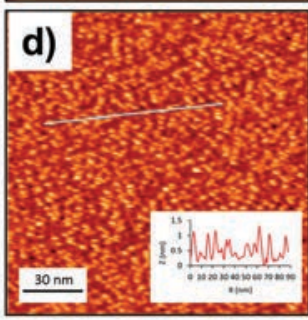

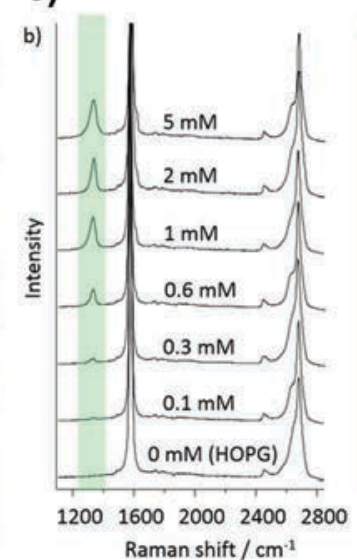

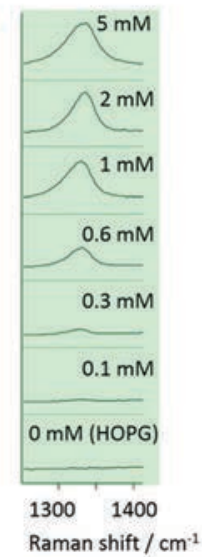

h)

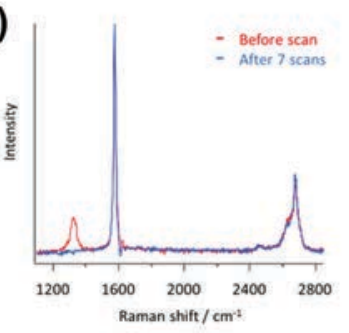

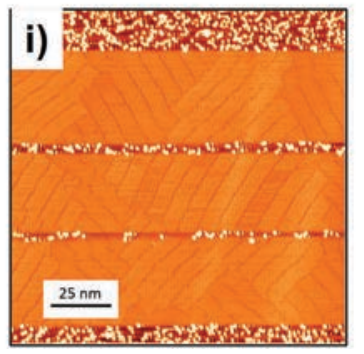

1

10

15

Fig. 17 (a) A schematic showing the attachment of phenyl radicals to the graphene/graphite surface using electrochemical (EC) reduction of diazonium salts. (b) A scheme showing the difference in the covalent chemistry of 4-NBD versus 3,5-TBD. (c and d) STM images showing the surface morphology after covalent grafting of 4-NBD (c), and 3,5-TBD (d) using $1 \mathrm{mM}$ solutions. (e) Raman spectra showing the evolution of the D band as function of concentration of 3,5-TBD used for the electrolysis. (f) Schematic showing an STM nanolithography experiment where the detachment of phenyl groups was carried out in well-defined narrow stripes (grey) using STM tip. (g) Confocal Raman D-band map of the stripes. (h) Raman spectra before (red) and after (blue) STM lithography. (i) Self-assembly of pentacontane within the degrafted nanocorrals. Reproduced from ref. 72 with permission from the American Chemical Society. 
1 using an STM often influences the assembly process even when working with regular solid surfaces. Such effects are dramatically enhanced when the self-assembly occurs in nanoconfined space as recently demonstrated in case of an alkylated diacetylene derivative. ${ }^{73}$

An increasing number of researchers have now started to combine supramolecular chemistry on surfaces with interfacial covalent chemistry. Recently a strategy combining physisorption and chemisorption was employed for exercising spatial 10 control over covalent attachment of diazonium radicals onto a graphene surface. ${ }^{74}$ The approach involved pre-assembly of an alkyl diazonium salt on the surface followed by electrochemical activation to yield aryl radicals which then covalently attach to the surface of graphene. The long alkyl chains attached to the 15 phenyldiazonium moiety facilitate the self-assembly of molecules into a columnar structure and this self-assembled network is then 'imprinted' into the covalent pattern by electrochemical activation. Similar strategies, where a supramolecular assembly step precedes the covalent chemistry, are rapidly evolving. ${ }^{75}$

\section{Recent advances: imaging and beyond imaging}

Since its inception in the 1980s both AFM as well as STM have evolved significantly, both in terms of the technology behind the methods as well as the applicability of the techniques to wide ranging issues. Originally intended for surface imaging, scanning probe microscopy has developed into versatile technique which can provide valuable information on electronic as well as mechanical properties of surface adsorbed material. Although invented in late 1980s, non-contact AFM (nc-AFM) has surpassed STM in terms of lateral resolution. The decoration of AFM tips with carbon monoxide molecules, which enabled first high-resolution nc-AFM images of pentacene, ${ }^{76}$ is now increasingly employed for high-resolution imaging of molecular adsorbates under ultrahigh vacuum conditions. The technique has already been applied for imaging of hydrogen-bonded arrays $^{77,78}$ (Fig. 18), for bond order discrimination, ${ }^{79}$ as well
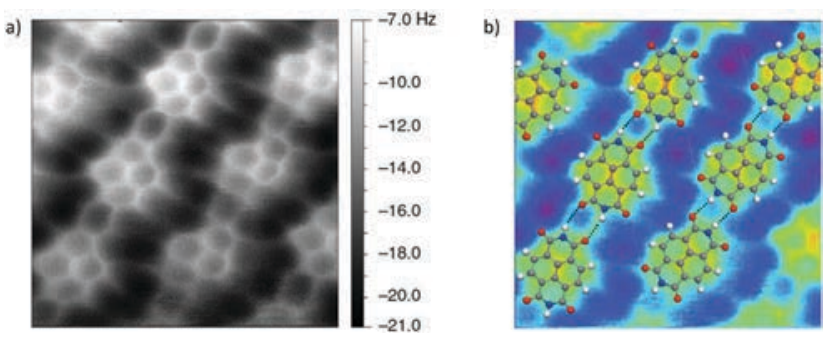

Fig. 18 (a) Constant height nc-AFM image of a hydrogen-bonded array of 1,4,5,8-naphthalenetetracarboxylic diimide (NTCDI) molecules on a Ag:Si(111) $-(\sqrt{ } 3 \times \sqrt{ } 3) R 30^{\circ}$ substrate acquired at $77 \mathrm{~K}$. (b) Overlay of a NTCDI model on a contrast-adjusted section of the image shown in (a). The intermolecular $\mathrm{H}$ bonds $(\mathrm{N}-\mathrm{H} \ldots \mathrm{O})$ are shown as dotted black lines.

55 Reproduced from ref. 78, with permission from the Nature Publishing Group. as for following chemical transformations down to atomic detail. ${ }^{80}$ A recent development on this front is the ability to measure mechanical properties of individual conformers of large organic molecules. The forces required to push two conformers of tetra(4-bromophenyl)porphyrin along $\mathrm{Cu}(111)$ surface were measured using nc-AFM measurements. Despite having comparable binding energies, the mechanochemical response of the two conformers was found to be significantly different. The conformational variation leads to significant differences in the diffusion barrier for the two conformers thus modifying the mechanical response of the two molecules towards lateral manipulation. ${ }^{81}$

Since nc-AFM provides resolution comparable to or better than STM, imaging of molecular adsorbates on insulating surfaces has become possible. Such surfaces offer a fundamentally different platform for molecular self-assembly. This is because the contribution of long-range repulsive interactions to molecular ordering is expected to be significant on insulating surfaces. Such repulsive interactions are often screened on conductive substrates yet self-assembly based on long-range repulsive interactions has been reported for molecules possessing a permanent electrical dipole moment as well as those which do not possess a permanent dipole moment. Calcite is one such insulating surface which enhances the repulsive interactions. For example, self-assembly of 3-hydroxybenzoic acid on calcite leads to formation of equidistant stripes that consist of hydrogen-bonded molecular double rows. Coveragedependent nc-AFM images revealed that the periodicity of the striped pattern changes depending on the surface coverage of molecules. This coverage-dependent periodicity is a strong indication of long-range repulsive interactions which at lower surface coverages manifests as molecular stripes that are spaced apart by $16 \mathrm{~nm}$. Since substrate templating as well as charge screening effects are either minimal or absent on insulating surfaces, this unique self-assembling pattern was explained using a generic model wherein the electrostatic repulsion originates solely from adsorption-induced dipoles. ${ }^{82}$

Imaging on insulating surfaces, especially at solution-solid interface using STM is not as straightforward. This prohibits the use of STM as a spectroscopic tool for probing the local density of electronic states and the band gap of surfaces and molecular adsorbates. This is typically achieved by monitoring either the current-voltage $(I-V)$ characteristics or the tunneling conductance $(\mathrm{d} I / \mathrm{d} V)$. However, a typical STM experiment requires a conducting surface and the molecular adsorbate is often electronically coupled with it. Thus, probing the intrinsic electronic properties of molecular adsorbates is not trivial and requires its electronic decoupling from the surface. Such decoupling is typically accomplished by introducing a very thin non-conductive layer between the substrate and the molecular layer. A thin $\mathrm{NaCl}$ layer is commonly used as an insulating layer in studies carried out under UHV conditions. The solutionsolid interface however proves much more challenging for such studies, due to poor stability of such layers and in view of inplane and out-of-plane molecular dynamics present under such conditions. A recent breakthrough in electronic decoupling of 
1 molecular layers at the solution-solid interface may prove to be a general approach for precise estimation of local spectroscopic properties. This approach involves use of self-assembled monolayer of triacontane as an insulating spacer between a gold surface and the STM tip. Long chain alkanes are known to self-assemble with their long axis parallel to the surface to form ordered layers. Such a layer of non-conductive molecules is thin enough to allow electrons to tunnel through, while effectively screening molecule-substrate interactions. Silicone oil was used 10 as a high-viscous liquid medium which significantly limits the degree of dynamics. This unique combination of spacer and the viscous medium allowed precise spectroscopic measurements on electronically decoupled single $\mathrm{C}_{60}$ molecules as well as flakes of graphene, providing detailed information on their molecular energy levels with high spatial and energy resolution. ${ }^{83}$

Embracing non-conductive surfaces is becoming a trend as it adds another dimension to the applicability of scanning probe methods allowing investigation of new materials and physical properties. Molecular self-assembly on insulating sur-

20 faces also provides opportunities to investigate the optical properties of supramolecular networks. Self-assembly of 5,10,15,20-tetrakis(4-carboxylphenyl)porphyrin (TCPP) was studied on the surface of hexagonal boron nitride (hBN). ${ }^{84}$ Remarkably, AFM imaging under ambient conditions provided sub-nanometer resolution of the supramolecular network allowing detailed molecular level characterization. The molecules form an ordered hydrogen-bonded array of either a hexagonal or a square lattice, the latter being more stable. Dispersion corrected DFT calculations revealed that the molecules undergo a bowing distortion upon adsorption on the hBN surface. The aryl side groups hinder good van der Waals contact with the underlying surface. The distortion in the molecular frame allows maximization of van der Waals interactions. This distortion is reflected in the fluorescence spectrum of the selfassembled network which was found to be red-shifted by $\sim 30 \mathrm{~nm}$ with respect to that in solution. The insulating nature of the hBN allows evaluation of such optical properties as selfassembled networks of TCPP adsorbed on conductive surfaces such as HOPG and $\mathrm{MoS}_{2}$ did not show any fluorescence.

An area where molecular self-assembly on surfaces is potentially powerful is in electronics, where molecular adsorption, be it physisorption or chemisorption modifies the substrates properties in a well-defined manner. The advantage of substrate functionalization based on molecular self-assembly ver45 sus spatially random adsorption is two-fold. The surface density and spatial ordering of a functional group can very efficiently be controlled by molecular self-assembly. We already hinted to the fact that spatially controlled grafting is of interest for controlling the band gap of graphene. But, additionally, non-covalent modification of graphite and in particular graphene is a powerful approach. One current key challenge in graphene research is to tune its charge carrier concentration, i.e., $\mathrm{p}$ - and n-type doping of graphene. Using the supramolecular self-assembly approach using alkyl amines that have varying chain lengths, tuneable n-type doping of graphene was obtained. ${ }^{85}$ The doping magnitude is modulated by controlling the density of the strong n-type doping amine groups on the surface. As revealed by scanning tunneling and atomic force microscopy, this density is governed by the length of the alkyl chain which acts as a spacer within the self-assembled network. The modulation of the doping magnitude depending on the chain length was demonstrated using Raman spectroscopy and electrical measurements on graphene field effect devices.

Another interesting development is the controlled formation of multilayers of the same material or stacks of different materials. Once could argue that physisorbed two-dimensional supramolecular assemblies are too weak to play a meaningful role. This is to a certain extent true but one should take great care not to generalize the "weakness" of these type of monolayers. As discussed, some systems show significant kinetic blockades reducing or eliminating the desorption of adsorbed molecules, even in a liquid environment. Recently, it was documented that ALD growth of Hf02 could be templated on a physisorbed PTCDA monolayer on epitaxial graphene on $\mathrm{SiC}(0001) .{ }^{86}$ In contrast to graphene itself, the chemical functionality provided by the PTCDA seeding layer resulted a homogeneous ALD layer.

\section{Conclusions}

The field of 2D supramolecular chemistry and surface selfassembly continues to grow and offers many exciting opportunities for future advances. As the field diversifies many different facets of research are the focus of recent studies. These include the development of increased understanding of the fundamental thermodynamic properties that underpin the surface-based self-assembly processes. As with solution phase supramolecular chemistry both enthalpic and entropic factors are significant but in the case of surface self-assembly the additional role of the surface is highly significant. The field is moving towards more applied investigations with an increasing emphasis on preparing chemically and physically robust systems. The increasing exploitation of covalent coupling reactions to prepare extended arrays or to modify existing structures is an emerging theme that we anticipate will continue to grow.

In contrast to other areas of supramolecular chemistry, the methods of characterisation employed in the field, notably scanning probe microscopies, allow a remarkable depth of understanding that facilitates the study of highly complex systems. Thus, recent studies have developed methods of assembling many component systems in a single step and also the assembly of highly complex prototypical fractal and quasicrystalline systems. In many ways, such systems lie at the pinnacle of supramolecular chemistry in terms of their complexity and this is made possible by the techniques and approaches that are made necessary by working on surfaces.

\section{Acknowledgements}

KSM and SDF thank the Fund of Scientific Research Flanders (FWO), Internal Funds KU Leuven, Belgian Federal Science Policy Office (IAP-7/05) for financial support. SDF acknowledges 
1 the support from the European Research Council under the European Union's Seventh Framework Programme (FP7/20072013)/ERC Grant Agreement No. 340324. NRC and NP thank the Engineering and Physical Sciences Research Council (EP/ N033906/1) for financial support. NRC gratefully acknowledges a Royal Society Wolfson Merit Award.

\section{References}

1 C. J. Pedersen, J. Am. Chem. Soc., 1967, 89, 7017-7036.

2 J. W. Steed and J. L. Atwood, Supramolecular Chemistry, John Wiley \& Sons, Ltd, 2009, pp. i-xxxi, DOI: 10.1002/ 9780470740880.fmatter.

3 G. R. Desiraju, J. Am. Chem. Soc., 2013, 135, 9952-9967.

4 J. A. A. W. Elemans, S. Lei and S. De Feyter, Angew. Chem., Int. Ed., 2009, 48, 7298-7332.

5 L. Xu, W. Ma, L. Wang, C. Xu, H. Kuang and N. A. Kotov, Chem. Soc. Rev., 2013, 42, 3114-3126.

6 A. G. Slater, P. H. Beton and N. R. Champness, Chem. Sci., 2011, 2, 1440-1448.

7 A. Kumar, K. Banerjee and P. Liljeroth, Nanotechnology, 2017, 28, 082001.

8 X. Bouju, C. Mattioli, G. Franc, A. Pujol and A. Gourdon, Chem. Rev., 2017, 117, 1407-1444.

9 S. M. Barlow and R. Raval, Surf. Sci. Rep., 2003, 50, 201-341.

10 P. Muller-Buschbaum, Polym. J., 2013, 45, 34-42.

11 T. Arnold and S. M. Clarke, Curr. Opin. Colloid Interface Sci., 2012, 17, 23-32.

3012 D. Shechtman, I. Blech, D. Gratias and J. W. Cahn, Phys. Rev. Lett., 1984, 53, 1951-1953.

13 N. A. Wasio, R. C. Quardokus, R. P. Forrest, C. S. Lent, S. A. Corcelli, J. A. Christie, K. W. Henderson and S. A. Kandel, Nature, 2014, 507, 86-89.

14 J. I. Urgel, D. Écija, G. Lyu, R. Zhang, C.-A. Palma, W. Auwärter, N. Lin and J. V. Barth, Nat. Chem., 2016, 8, 657-662.

15 J. Shang, Y. Wang, M. Chen, J. Dai, X. Zhou, J. Kuttner, G. Hilt, X. Shao, J. M. Gottfried and K. Wu, Nat. Chem., 2015, 7, 389-393.

16 A. Rastgoo-Lahrood, N. Martsinovich, M. Lischka, J. Eichhorn, P. Szabelski, D. Nieckarz, T. Strunskus, K. Das, M. Schmittel, W. M. Heckl and M. Lackinger, ACS Nano, 2016, 10, 10901-10911.

17 X. Zhang, N. Li, G.-C. Gu, H. Wang, D. Nieckarz, P. Szabelski, Y. He, Y. Wang, C. Xie, Z.-Y. Shen, J.-T. Lü, H. Tang, L.-M. Peng, S.-M. Hou, K. Wu and Y.-F. Wang, ACS Nano, 2015, 9, 11909-11915.

18 Q. Sun, L. Cai, H. Ma, C. Yuan and W. Xu, Chem. Commun., 2015, 51, 14164-14166.

19 X. Zhang, N. Li, L. Liu, G. Gu, C. Li, H. Tang, L. Peng, S. Hou and Y. Wang, Chem. Commun., 2016, 52, 10578-10581.

20 G. Gu, N. Li, L. Liu, X. Zhang, Q. Wu, D. Nieckarz, P. Szabelski, L. Peng, B. K. Teo, S. Hou and Y. Wang, RSC $A d v .$, 2016, 6, 66548-66552.

21 U. Mazur and K. W. Hipps, Chem. Commun., 2015, 51, 4737-4749.
22 A. Bhattarai, U. Mazur and K. W. Hipps, J. Am. Chem. Soc., 2014, 136, 2142-2148.

23 A. Bhattarai, U. Mazur and K. W. Hipps, J. Phys. Chem. C, 2015, 119, 9386-9394.

24 A. Bellec, C. Arrigoni, G. Schull, L. Douillard, C. FioriniDebuisschert, F. Mathevet, D. Kreher, A.-J. Attias and F. Charra, J. Chem. Phys., 2011, 134, 124702.

25 R. A. Barnard and A. J. Matzger, Langmuir, 2014, 30, 7388-7394.

26 W. Song, N. Martsinovich, W. M. Heckl and M. Lackinger, J. Am. Chem. Soc., 2013, 135, 14854-14862.

27 M. Mammen, E. I. Shakhnovich and G. M. Whitesides, J. Org. Chem., 1998, 63, 3168-3175.

28 S. Yokoyama, T. Hirose and K. Matsuda, Chem. Commun., 2014, 50, 5964-5966.

29 B. A. Friesen, A. Bhattarai, U. Mazur and K. W. Hipps, J. Am. Chem. Soc., 2012, 134, 14897-14904.

30 S. Griessl, M. Lackinger, M. Edelwirth, M. Hietschold and W. Heckl, Single Mol., 2002, 3, 25-31.

31 K. Cui, F. Schlütter, O. Ivasenko, M. Kivala, M. G. Schwab, S.-L. Lee, S. F. L. Mertens, K. Tahara, Y. Tobe, K. Müllen, K. S. Mali and S. De Feyter, Chem. - Eur. J., 2015, 21, 1652-1659.

32 A. G. Slater, L. M. A. Perdigao, P. H. Beton and N. R. Champness, Acc. Chem. Res., 2014, 47, 3417-3427.

33 J. Teyssandier, S. D. Feyter and K. S. Mali, Chem. Commun., 2016, 52, 11465-11487.

34 Y. Xue and M. Zimmt, J. Am. Chem. Soc., 2012, 134, 4513-4516. 35 J. Adisoejoso, K. Tahara, S. Okuhata, S. Lei, Y. Tobe and S. De Feyter, Angew. Chem., Int. Ed., 2009, 48, 7353-7357.

36 G. Velpula, T. Takeda, J. Adisoejoso, K. Inukai, K. Tahara, K. Mali, Y. Tobe and S. De Feyter, Chem. Commun., 2017, 53, 1108-1111.

37 M. Blunt, X. Lin, M. C. Gimenez-Lopez, M. Schröder, N. R. Champness and P. H. Beton, Chem. Commun., 2008, 2304-2306.

38 J. Liu, T. Chen., X. Deng, D. Wang, J. Pei and L.-J. Wan, J. Am. Chem. Soc., 2011, 133, 21010-21015.

39 R. Raval, Chem. Soc. Rev., 2009, 38, 707-721.

40 E. Gomar-Nadal, J. Puigmarti-Luis and D. B. Amabilino, Chem. Soc. Rev., 2008, 37, 490-504.

41 M. M. Green, N. C. Peterson, T. Sato, A. Teramoto, R. Cook and S. Lifson, Science, 1995, 268, 1860-1866.

42 E. Ghijesens, H. Cao, A. Noguchi, O. Ivasenko, Y. Fang, K. Tahara, Y. Tobe and S. De Feyter, Chem. Commun., 2015, 51, 4766-4769.

43 K. Tahara, H. Yamaga, E. Ghijsens, K. Inukai, J. Adisoejoso, M. O. Blunt, S. De Feyter and Y. Tobe, Nat. Chem., 2011, 3, 714-719.

44 Y. Fang, E. Ghijsens, O. Ivasenko, H. Cao, A. Noguchi, K. S. Mali, K. Tahara, Y. Tobe and S. De Feyter, Nat. Chem., 2016, 8, 711-717.

45 T. Chen, W.-H. Yang, D. Wang and L.-J. Wan, Nat. Commun., 2013, 4, 1389.

46 T. Chen, S.-Y. Li, D. Wang, M. Yao and L.-J. Wan, Angew. Chem., 2015, 127, 4383-4388.

47 R. Gutzler, T. Sirtl, J. F. Dienstmaier, K. Mahata, W. M. Heckl, M. Schmittel and M. Lackinger, J. Am. Chem. Soc., 2010, 132, 5084-5090. 
48 P. A. Staniec, L. M. A. Perdigão, A. Saywell, N. R. Champness and P. H. Beton, ChemPhysChem, 2007, 8, 2177-2181.

49 M. O. Blunt, J. Adisoejoso, K. Tahara, K. Katayama, M. Van der Auweraer, Y. Tobe and S. De Feyter, J. Am. Chem. Soc., 2013, 135, 12068-12075.

50 Y.-T. Shen, K. Deng, X.-M. Zhang, W. Feng, Q.-D. Zeng, C. Wang and J. R. Gong, Nano Lett., 2011, 11, 3245-3250.

51 K. Tahara, K. Inukai, J. Adisoejoso, H. Yamaga, T. Balandina, M. O. Blunt, S. De Feyter and Y. Tobe, Angew. Chem., Int. Ed., 2013, 52, 8373-8376.

52 N. Maeda, T. Hirose, S. Yokoyama and K. Matsuda, J. Phys. Chem. C, 2016, 120, 9317-9325; S. Yokoyama, T. Hirose and K. Matsuda, Langmuir, 2015, 31, 6404-6414; T. Sakano, Y. Imaizumi, T. Hirose and K. Matsuda, Chem. Lett., 2013, 42, 1537-1539.

53 S. Bonacchi, M. El Garah, A. Ciesielski, M. Herder, S. Conti, M. Cecchini, S. Hecht and P. Samori, Angew. Chem., Int. Ed., 2015, 54, 4865-4869.

54 Q.-N. Zheng, X.-H. Liu, X.-R. Liu, T. Chen, H.-J. Yan, Y.W. Zhong, D. Wang and L.-J. Wan, Angew. Chem., Int. Ed., 2014, 53, 13395-13399.

55 M. O. Blunt, J. C. Russell, M. C. Gimenez-Lopez, N. Taleb, X. Lin, M. Schröder, N. R. Champness and P. H. Beton, Nat. Chem., 2011, 3, 74-78.

56 F. P. Cometto, K. Kern and M. Lingenfelder, ACS Nano, 2015, 5, 5544-5550.

57 D. den Boer, M. Li, T. Habets, P. Iavicoli, A. E. Rowan, R. J. M. Nolte, S. Speller, D. B. Amabilino, S. De Feyter and J. A. A. W. Elemans, Nat. Chem., 2013, 5, 621-627.

3058 Y. Okawa and M. Aono, J. Chem. Phys., 2001, 115, 2317-2322.

59 Y. Okawa, S. K. Mandal, C. Hu, Y. Tateyama, S. Goedecker, S. Tsukamoto, T. Hasegawa, J. K. Gimzewski and M. Aono, J. Am. Chem. Soc., 2011, 133, 8227-8233.

60 M. Nakaya, Y. Okawa, C. Joachim, M. Aono and T. Nakayama, ACS Nano, 2014, 8, 12259-12264.

61 L. Colazzo, F. Sedona, A. Moretto, M. Casarin and M. Sambi, J. Am. Chem. Soc., 2016, 138, 10151-10156.

62 J. Eichhorn, W. M. Heckl and M. Lackinger, Chem. Commun., 2013, 49, 2900-2902; H.-Y. Gao, H. Wagner, D. Zhong, J.-H. Franke, A. Studer and H. Fuchs, Angew. Chem., Int. Ed., 2013, 52, 4024-4028; A. Saywell, A. S. Browning, P. Rahe, H. L. Anderson and P. H. Beton, Chem. Commun., 2016, 52, 10342-10345.

63 L. Lafferentz, V. Eberhardt, C. Dri, C. Africh, G. Comelli, F. Esch, S. Hecht and L. Grill, Nat. Chem., 2012, 4, 215-220; M. O. Blunt, J. C. Russell, N. R. Champness and P. H. Beton, Chem. Commun., 2010, 46, 7157-7159; H. Zhang, Z. Gong, K. Sun, R. Duan, P. Ji, L. Li, C. Li, K. Müllen and L. Chi, J. Am. Chem. Soc., 2016, 138, 11743-11748.

64 J. F. Dienstmaier, A. M. Gigler, A. J. Goetz, P. Knochel, T. Bein, A. Lyapin, S. Reichlmaier, W. M. Heckl and M. Lackinger, ACS Nano, 2011, 5, 9737-9745.

65 J. F. Dienstmaier, D. D. Medina, M. Dogru, P. Knochel, T. Bein, W. M. Heckl and M. Lackinger, ACS Nano, 2012, 6, 7234-7242. 13771-13774.

67 C.-Z. Guan, D. Wang and L.-J. Wan, Chem. Commun., 2012, 48, 2943-2945.

68 L. Xu, X. Zhou, Y. Yu, W. Q. Tian, J. Ma and S. Lei, ACS Nano, 2013, 7, 8066-8073.

69 G. L. C. Paulus, Q. H. Wang and M. S. Strano, Acc. Chem. Res., 2013, 46, 160-170.

70 K. C. Knirsch, N. C. Berner, H. C. Nerl, C. S. Cucinotta, Z. Gholamvand, N. McEvoy, Z. Wang, I. Abramovic, P. Vecera, M. Halik, S. Sanvito, G. S. Duesberg, V. Nicolosi, F. Hauke, A. Hirsch, J. N. Coleman and C. Backes, ACS Nano, 2015, 9, 6018-6030.

71 K. S. Mali, J. Greenwood, J. Adisoejoso, R. Phillipson and S. De Feyter, Nanoscale, 2015, 7, 1566-1585.

72 J. Greenwood, T. H. Phan, Y. Fujita, Z. Li, O. Ivasenko, W. Vanderlinden, H. Van Gorp, W. Frederickx, G. Lu, K. Tahara, Y. Tobe, H. Uji-i, S. F. L. Mertens and S. De Feyter, ACS Nano, 2015, 9, 5520-5535.

73 L. Verstraete, J. Greenwood, B. E. Hirsch and S. De Feyter, ACS Nano, 2016, 10, 10706-10715.

74 Z. Xia, F. Leonardi, M. Gobbi, Y. Liu, V. Bellani, A. Liscio, A. Kovtun, R. Li, X. Feng, E. Orgiu, P. Samorì, E. Treossi and V. Palermo, ACS Nano, 2016, 10, 7125-7134.

75 V. Q. Nguyen, X. Sun, F. Lafolet, J.-F. Audibert, F. Miomandre, G. Lemercier, F. Loiseau and J.-C. Lacroix, J. Am. Chem. Soc., 2016, 138, 9381-9384.

76 L. Gross, F. Mohn, N. Moll, P. Liljeroth and G. Meyer, Science, 2009, 325, 1110-1114.

77 J. Zhang, P. Chen, B. Yuan, W. Ji, Z. Cheng and X. Qiu, Science, 2013, 342, 611-614.

78 A. M. Sweetman, S. Jarvis, H. Sang, I. Lekkas, P. Rahe, Y. Wang, J. Wang, N. R. Champness, L. Kantorovich and P. J. Moriarty, Nat. Commun., 2014, 5, 3931.

79 L. Gross, F. Mohn, N. Moll, B. Schuler, A. Criado, E. Guitián, D. Peña, A. Gourdon and G. Meyer, Science, 2012, 337, 1326-1329.

80 S. Kawai, V. Haapasilta, B. D. Lindner, K. Tahara, P. Spijker, J. A. Buitendijk, R. Pawlak, T. Meier, Y. Tobe, A. S. Foster and E. Meyer, Nat. Commun., 2016, 7, 12711.

81 S. P. Jarvis, S. Taylor, J. D. Baran, N. R. Champness, J. A. Larsson and P. Moriarty, Nat. Commun., 2015, 6, 8338.

82 J. L. Neff, H. Söngen, R. Bechstein, P. Maass and A. Kühnle, J. Phys. Chem. C, 2015, 119, 24927-24931.

83 P. Nirmalraj, D. Thompson, A. Molina-Ontoria, M. Sousa, N. Martín, B. Gotsmann and H. Riel, Nat. Mater., 2014, 13, 947-953.

84 V. V. Korolkov, S. A. Svatek, A. Summerfield, J. Kerfoot, L. Yang, T. Taniguchi, K. Watanabe, N. R. Champness, N. A. Besley and P. H. Beton, ACS Nano, 2015, 9, 10347-10355.

85 R. Phillipson, C. J. Lockhart de la Rosa, J. Teyssandier, P. Walke, D. Waghray, Y. Fujita, J. Adisoejoso, K. S. Mali, I. Asselberghs, C. Huyghebaert, H. Uji-i, S. De Gendt and S. De Feyter, Nanoscale, 2016, 8, 20017-20026.

86 J. M. P. Alaboson, Q. H. Wang, J. D. Emery, A. L. Lipson, M. J. Bedzyk, J. W. Elam, M. J. Pellin and M. C. Hersam, ACS Nano, 2011, 5, 5223-5232.
1 